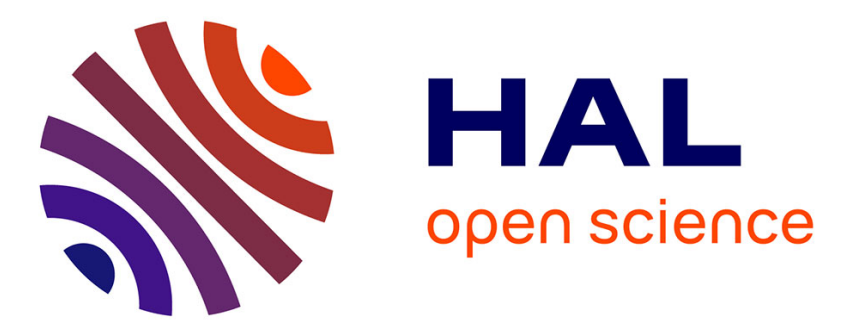

\title{
Heat Kernel coupled with geometric flow, and Ricci flow
} Koléhè Abdoulaye Coulibaly-Pasquier

\section{To cite this version:}

Koléhè Abdoulaye Coulibaly-Pasquier. Heat Kernel coupled with geometric flow, and Ricci flow. Séminaire de Probabilités, 2019, L, pp.221-256. 10.1007/978-3-030-28535-7_11 . hal-00767995v2

\section{HAL Id: hal-00767995 \\ https://hal.science/hal-00767995v2}

Submitted on 10 Oct 2019

HAL is a multi-disciplinary open access archive for the deposit and dissemination of scientific research documents, whether they are published or not. The documents may come from teaching and research institutions in France or abroad, or from public or private research centers.
L'archive ouverte pluridisciplinaire HAL, est destinée au dépôt et à la diffusion de documents scientifiques de niveau recherche, publiés ou non, émanant des établissements d'enseignement et de recherche français ou étrangers, des laboratoires publics ou privés.

\section{(ㄷ)(1)}

Distributed under a Creative Commons Attribution| 4.0 International License 


\title{
HEAT KERNEL COUPLED WITH GEOMETRIC FLOW, AND RICCI FLOW
}

\author{
KOLÉHÈ A. COULIBALY-PASQUIER
}

\begin{abstract}
We prove on-diagonal upper bound for the minimal fundamental solution of the heat equation evolving under geometric flow. In the case of Ricci flow, with non-negative Ricci curvature and a condition on the growth of volume of ball for the initial manifold, we derive Gaussian bounds for the minimal fundamental solution of the heat equation, and then for the conjugate heat equation.
\end{abstract}

\section{INTRODUCTION}

Let $(M, g(t))$ be a complete Riemannian manifold, either non compact or compact without boundary, $g(t)$ be a family of metrics on $M, \nabla^{g(t)}$ and $\Delta_{g(t)}$ the corresponding gradient and Laplace-Beltrami operator, $\mathrm{Ric}_{g(t)}$ the corresponding Ricci curvature, $\mu_{g(t)}$ the corresponding Riemannian volume, $d_{g(t)}(x, y)$ be the distance function, and $B_{g(t)}(x, r)$ the geodesic ball of radius $r$ for the distance $d_{g(t)}$. Sometimes to reduce the notation, when there are no risk of confusion concerning the family of metric we simply write $\nabla^{t}, \Delta_{t}$, $\mu_{t}, \ldots$

Let $\alpha_{i, j}(t)$ be a family of symmetric 2 -tensors on $M$. We consider the following heat equation coupled with a geometric flow.

$$
\left\{\begin{array}{l}
\partial_{t} g_{i, j}(t)=\alpha_{i, j}(t), \\
\partial_{t} f(t, x)=\frac{1}{2} \Delta_{t} f(t, x), \\
f(0, x)=f_{0}(x) .
\end{array}\right.
$$

We are interested in estimating the minimal fundamental solution of (1.1). For the existence of minimal fundamental solution in non compact case we refer to Chapter 24 of [9]. An estimate of this fundamental solution, already give an estimate of the conjugate heat equation, which is the density of the $g(t)$-Brownian motion introduced in $[1,10]$ see also [11]. Moreover estimate of the fundamental solution of the heat equation have many geometric applications, both in constant metric case and geometric flows for instance in $[21,8,5,23]$.

Such a flow, have been investigated in the literature. We mention the following situations.

- The most famous case is when $\alpha_{i, j}(t):=0$. This is the case of constant metric and equation (1.1) is the usual heat equation in $M$. 
- The Ricci flow corresponds to $\alpha(t):=-\operatorname{Ric}_{g(t)}$.

- We can also consider $\alpha_{i, j}(t):=-2 h H_{i, j}(g(t))$, where $H_{i, j}(g(t))$ is the second fundamental form according to the metric $g(t)$, and $h$ is the mean curvature, when the family of metric derives from the mean curvature flow.

The existence of the Ricci flow $\partial_{t} g(t)=-\operatorname{Ric}_{g(t)}$ for compact manifold was proved in [18]. Under additional assumptions, the existence of the Ricci flow for a complete manifold was proved in [24]. For the last example, the existence result of the mean curvature flow for a compact manifold could be found in [12].

Using stochastic calculus we prove on-diagonal upper bound for the minimal fundamental solution of the heat equation (1.1), for general geometric flow. As far as we know this result is new. We derive a Gaussian upper bound for the minimal heat kernel coupled with the Ricci flow, in the case of positive Ricci curvature and condition on the growth of volume of ball for the initial manifold (i.e Hypothesis H1 in Theorem 4.8).

Related results. Previous stochastic proof of Harnack inequality with power appear in [3] for the constant metric case. Note that our coupling is different from the coupling in [3], and simplify the argument since we do not need to take care of different cutlocus. The Harnack inequality with power we obtain also appears in [7], and is obtain by different way.

For the Ricci flow, Gaussian upper bounds could be found as example in [23] where the author use Harnack inequality and doubling volume property. An over one by Zhang and Cao [5] uses Sobolev type inequality that is conserved along Ricci flow.

Outline. The paper is organized as follows. In section 2 we define a horizontal coupling. We use this coupling and Girsanov's Theorem in order to generalize Harnack inequality with power -for inhomogeneous heat equation - introduced by F.Y Wang [25] see also [14, 3]. We also use this coupling to give some isoperimetric-type Harnack inequality in Lemma 2.7 and ultracontractivity of the heat kernel in Corollary 2.11.

In Section 3, since the heat kernel of (1.1) is in general non symmetric, the Gaussian bound is not a direct consequence of Harnack inequality with power as in [14]. To overcome this difficulty we use the dual process and derive on-diagonal upper estimate of the heat kernel of (1.1) in Theorem 3.1 .

The Section 4 is devoted to the case of Ricci flow. We use modification of Grigor'yan trick to derive Gaussian Heat kernel bounds from the ondiagonal upper bound. The principal result of this section is Theorem 4.8 and Corollary 4.9.

\section{Coupling and Harnack inequality with POWer}

2.1. Coupling. In the first part of this section, we focus on the operator of type $L_{t}:=\frac{1}{2} \Delta_{g(t)}$, where $\Delta_{g(t)}$ is the Laplace operator associated to a 
time dependent family of metrics $g(t)_{t \in\left[0, T_{c}\right.}$. We suppose that $(M, g(t))$ is complete for all $t \in\left[0, T_{c}\right.$. Let $x \in M$ and $t \mapsto X_{t}(x)$ be the $g(t)$-Brownian motion started at $x$. The notion of $g(t)$-Brownian motion, i.e. a $L_{t}$ diffusion, parallel transport, and damped parallel transport has been given in $[10,1]$. We also suppose in this section that all $g(t)$-Brownian motion is non-explosive (i.e. stochastically complete).

Since we use different family of metrics all construction depends on the family of metrics.

Let $/ /_{t}^{g(t), X .(x)}$ be the $g(t)$ parallel transport above $t \mapsto X_{t}(x)$, which is a linear isometry

$$
\begin{aligned}
/_{t}^{g(t), X .(x)} & : \quad\left(T_{x} M, g(0)\right) \longrightarrow\left(T_{X_{t}(x)} M, g(t)\right) \\
/ /_{0}^{g(t), X .(x)} & =I d_{T_{x} M}
\end{aligned}
$$

Let $\mathbf{W}_{t}^{g(t), X .(x)}$ be the damped parallel transport that satisfies the following Stratonovich covariant equation:

$$
\begin{aligned}
& * d\left(/ /_{t}^{g(t), X .(x)}\right)^{-1}\left(\mathbf{W}_{t}^{g(t), X .(x)}\right) \\
& =-\frac{1}{2}\left(/ /_{t}^{g(t), X .(x)}\right)^{-1}\left(\operatorname{Ric}_{g(t)}-\partial_{t} g(t)\right)^{\# g(t)}\left(\mathbf{W}_{0, t}^{g(t), X .(x)}\right) d t .
\end{aligned}
$$

It is a linear operator between:

$$
\begin{aligned}
& \mathbf{W}_{t}^{g(t), X .(x)}: T_{x} M \longrightarrow T_{X_{t}(x)} M \\
& \mathbf{W}_{0}^{g(t), X .(x)}=\operatorname{Id}_{T_{x} M} .
\end{aligned}
$$

In [2] we give a construction of a process with value in a space of curves. Since we sometimes change the underlying family of metrics, we incorporate this family of metrics in the notation.

Let $x, y \in M, u \mapsto \gamma(u)$ be a $g(0)$ geodesic curve such that $\gamma(0)=x$ and $\gamma(1)=y$ and $t \mapsto\left(X_{t}(u)_{u \in[0,1]}\right)$ be the horizontal $L_{t}$-diffusion in $C^{1}$ path space $C^{1}([0,1], M)$ over $X_{t}(x)$ that starts at $\gamma$, where $X_{t}(x)$ is a $g(t)$ Brownian motion that starts at $x$. By assumption it is defined for all $t \in[0, T]$ with $T<T_{c}$.

We will recall the usual properties satisfied by the horizontal $L_{t}$-diffusion in $C^{1}$ path space Theorem $3.1[2]$ :

The family

$$
u \mapsto\left(X_{t}(u)\right)_{t \in[0, T]}
$$

is a family of $L_{t}$-diffusions. It is a.s. continuous in $(t, u)$ and $C^{1}$ in $u$, satisfies

$$
X_{t}(0)=X_{t}(x) \text { and } X_{0}(u)=\gamma(u),
$$

and solves the equation

$$
\partial_{u} X_{t}(u)=\mathbf{W}_{t}^{g(t), X(u)}(\dot{\gamma}(u)) .
$$


Furthermore, $X .(u)$ satisfies the following Itô stochastic differential equation

$$
d^{\nabla_{t}} X_{t}(u)=P_{0, u}^{g(t), X_{t}(.)} d^{\nabla_{t}} X_{t}(0),
$$

where

$$
P_{0, u}^{g(t), X_{t}(.)}: T_{X_{t}(0)} M \rightarrow T_{X_{t}(u)} M
$$

denotes usual parallel transport along the $C^{1}$-curve

$$
[0, u] \rightarrow M, \quad v \mapsto X_{t}(v)
$$

with respect to the metric $g(t)$.

We often use the notation $/ / t$ for $/ /_{t}^{g(t), X .(x)}$ when there no risk of confusion of the underling process and the family of metrics.

Proposition 2.1. Suppose that the $g(t)$-Brownian motion starting at $x$ is non-explosive. The diagonal process $t \mapsto X_{t}\left(\frac{t}{T}\right)$ satisfies the following stochastic differential equation:

$$
d^{\nabla_{t}}\left(X .\left(\frac{\dot{T}}{T}\right)\right)_{t}=P_{0, \frac{t}{T}}^{g(t), X_{t}(.)} d^{\nabla_{t}} X_{t}(0)+\frac{1}{T} \boldsymbol{W}_{t}^{g(t), X .\left(\frac{t}{T}\right)} \dot{\gamma}\left(\frac{t}{T}\right) d t
$$

Proof. We pass to the Stratonovich differential to obtain the following chain rule formula at time $t_{0}$ :

$$
* d\left(X .\left(\frac{\dot{\bar{T}}}{T}\right)\right)_{t_{0}}=* d\left(X .\left(\frac{t_{0}}{T}\right)\right)_{t_{0}}+\left.\frac{d X_{t_{0}}\left(\frac{t}{T}\right)}{d t}\right|_{t=t_{0}} d t_{0} .
$$

We use (2.1) to identify the last term of the right hand side:

$$
\left.\frac{d X_{t_{0}}\left(\frac{t}{T}\right)}{d t}\right|_{t=t_{0}}=\frac{1}{T} \mathbf{W}_{t_{0}}^{g(t), X \cdot\left(\frac{t_{0}}{T}\right)}\left(\dot{\gamma}\left(\frac{t_{0}}{T}\right)\right) .
$$

Now we come back to the Itô differential equation using the following relation:

$$
d^{\nabla_{t}} Y_{t}=/ /_{t}^{Y \cdot}\left(d \int_{0}^{t}\left(/ /_{s}^{Y^{*}}\right)^{-1} * d Y_{s}\right)
$$

and we obtain

$$
\begin{aligned}
& d^{\nabla_{t_{0}}}\left(X .\left(\frac{\dot{\bar{T}}}{T}\right)\right)_{t_{0}} \\
& =/ / t_{0}\left(d \int_{0}^{t_{0}} / /_{s}^{-1} * d\left(X \cdot\left(\frac{t_{0}}{T}\right)\right)_{s}+\frac{1}{T} / /_{s}^{-1} \mathbf{W}_{s}^{g(s), X \cdot\left(\frac{s}{T}\right)}\left(\dot{\gamma}\left(\frac{s}{T}\right)\right) d s\right) \\
& =d^{\nabla_{t_{0}}}\left(X \cdot\left(\frac{t_{0}}{T}\right)\right)_{t_{0}}+\frac{1}{T} \mathbf{W}_{t_{0}}^{g\left(t_{0}\right), X \cdot\left(\frac{t_{0}}{T}\right)} \dot{\gamma}\left(\frac{t_{0}}{T}\right) .
\end{aligned}
$$

We then use (2.2) to identify

$$
d^{\nabla t_{0}}\left(X .\left(\frac{t_{0}}{T}\right)\right)_{t_{0}}=P_{0, \frac{t_{0}}{T}}^{g\left(t_{0}\right), X_{t_{0}}(.)} d^{\nabla_{t_{0}}} X_{t_{0}}(0) .
$$

Thus concludes the proof. 
Let

$$
\begin{gathered}
N_{t}:=-\frac{1}{T} \int_{0}^{t}\left\langle P_{0, \frac{s}{T}}^{g(s), X_{s}(.)} d^{\nabla_{s}} X_{s}(0), \mathbf{W}_{s}^{g(s), X .\left(\frac{s}{T}\right)} \dot{\gamma}\left(\frac{s}{T}\right)\right\rangle_{g(s)}, \\
R_{t}:=\exp \left(N_{t}-\frac{1}{2}\langle N\rangle_{t}\right) .
\end{gathered}
$$

In many situations Novikov's criterion is satisfied. Therefore we could expect $R_{t}$ to be a martingale. Define the new probability measure $\mathbb{Q}$ as :

$$
\mathbb{Q}:=R_{T} \mathbb{P} \text {. }
$$

Proposition 2.2. Suppose that the $g(t)$-Brownian motion starting at $x$ is non-explosive and suppose that Novikov's criterion is satisfied for $N_{t}$. Then under $\mathbb{Q}$, the process $X_{t}\left(\frac{t}{T}\right)$ is a $L_{t}$-diffusion that starts at $x$, and finishes at $X_{T}(1)=X_{T}(y)$, i.e. under $\mathbb{Q}, X_{T}(1)$ have the same distribution as the $g(t)$-Brownian motion at time $T$ that start at $y$.

Proof. One could directly apply Girsanov's theorem. We prefer here to give a direct proof. Let $f \in C_{b}^{2}(M, \mathbb{R})$, since $N_{t}$ satisfy Novikov's condition $R_{t}$ is a $\mathbb{P}$-martingale. We use Itô formula to compute :

$$
\begin{aligned}
d f\left(X_{t}\left(\frac{t}{T}\right)\right) & =\left\langle\nabla^{t} f\left(X_{t}\left(\frac{t}{T}\right)\right), d^{\nabla_{t}}\left(X .\left(\frac{\dot{\bar{T}}}{T}\right)\right)_{t}\right\rangle_{g(t)} \\
& +\frac{1}{2} \operatorname{Hess}_{t} f\left(X_{t}\left(\frac{t}{T}\right)\right)\left(d^{\nabla_{t}}\left(X .\left(\frac{\dot{\bar{T}}}{T}\right)\right)_{t}, d^{\nabla_{t}}\left(X .\left(\frac{\dot{\bar{T}}}{T}\right)\right)_{t}\right) .
\end{aligned}
$$

Since $P_{0, \frac{t}{T}}^{g(t), X_{t}(.)}$ is an isometry for the metric $g(t)$

$$
\begin{aligned}
d f\left(X_{t}\left(\frac{t}{T}\right)\right) & =\left\langle\nabla^{t} f\left(X_{t}\left(\frac{t}{T}\right)\right), P_{0, \frac{t}{T}}^{g(t), X_{t}(.)} d^{\nabla_{t}} X_{t}(0)\right\rangle_{g(t)} \\
& +\frac{1}{T}\left\langle\nabla^{t} f\left(X_{t}\left(\frac{t}{T}\right)\right), \mathbf{W}_{t}^{g(t), X .\left(\frac{t}{T}\right)} \dot{\gamma}\left(\frac{t}{T}\right)\right\rangle_{g(t)} d t+\frac{1}{2} \Delta_{t} f\left(X_{t}\left(\frac{t}{T}\right)\right) d t .
\end{aligned}
$$

Moreover

$$
d R_{t} d\left(f\left(X_{t}\left(\frac{t}{T}\right)\right)\right)=-\frac{1}{T} R_{t}\left\langle\nabla^{t} f\left(X_{t}\left(\frac{t}{T}\right)\right), \mathbf{W}_{t}^{g(t), X .\left(\frac{t}{T}\right)} \dot{\gamma}\left(\frac{t}{T}\right)\right\rangle_{g(t)} d t .
$$

This implies

$$
d\left(R_{t} f\left(X_{t}\left(\frac{t}{T}\right)\right)\right)=\frac{1}{2} R_{t} \Delta_{t} f\left(X_{t}\left(\frac{t}{T}\right)\right) d t+d M_{t}^{\mathbb{P}},
$$

where $M_{t}^{\mathbb{P}}$ is a martingale for $\mathbb{P}$. On the other hand, since $R_{t}$ is a $\mathbb{P}$ martingale, we have

$$
R_{t} \int_{0}^{t} \Delta_{s} f\left(X_{s}\left(\frac{s}{T}\right)\right) d s=\int_{0}^{t} R_{s} \Delta_{s} f\left(X_{s}\left(\frac{s}{T}\right)\right) d s+\tilde{M}^{\mathbb{P}},
$$

where $\tilde{M}_{s}^{\mathbb{P}}$ is a $\mathbb{P}$-martingale.

Thus

$$
R_{t}\left(f\left(X_{t}\left(\frac{t}{T}\right)\right)-\frac{1}{2} \int_{0}^{t} \Delta_{s} f\left(X_{s}\left(\frac{s}{T}\right)\right) d s\right)
$$


is a martingale.

Since $U_{t}$ is a $\mathbb{Q}$-martingale if and only if $R_{t} U_{t}$ is a $\mathbb{P}$-martingale, $f\left(X_{t}\left(\frac{t}{T}\right)\right)$ $\frac{1}{2} \int_{0}^{t} \Delta_{s} f\left(X_{s}\left(\frac{s}{T}\right)\right) d s$ is then a $\mathbb{Q}$ martingale i.e. $X_{t}\left(\frac{t}{T}\right)$ is a $L_{t}$ diffusion under the probability $\mathbb{Q}$. It is clear that it finishes at $X_{T}(y)$. Thus $X_{t}\left(\frac{t}{T}\right)$ can be seen as a coupling between two $L_{t}$ diffusions that starts at different points up to changing probability.

2.2. Harnack inequality with power and some semigroup property. Let $T_{c}$ be the maximal life time of geometric flow $g(t)_{t \in\left[0, T_{c}[\right.}$. For all $T<$ $T_{c}$, let $X_{t}^{T}$ be a $g(T-t)$-Brownian motion and $/ /_{0, t}^{T}:=/ /_{0, t}^{g(T-t), X^{T}}$ be the associated parallel transport. In this case, for a solution $f(t,$.$) of (1.1)$, $f\left(T-t, X_{t}^{T}(x)\right)$ is a local martingale for any $x \in M$. Hence the following representation holds for the solution :

$$
P_{0, T} f_{0}(x):=f(T, x)=\mathbb{E}_{x}\left[f_{0}\left(X_{T}^{T}\right)\right] .
$$

The subscript $T$ refers to the fact that a time reversal step is involved.

Let $\mathbf{W}_{0, t}^{T}:=\mathbf{W}_{0, t}^{g(T-t), X^{T}}$ be the damped parallel transport along the $g(T-$ $t$ )-Brownian motion. We recall the covariant differential equation satisfied by this damped parallel transport (2.1):

$* d\left(\left(/ /_{0, t}^{T}\right)^{-1}\left(\mathbf{W}_{0, t}^{T}\right)\right)=-\frac{1}{2}\left(/ /_{0, t}^{T}\right)^{-1}\left(\operatorname{Ric}_{g(T-t)}-\partial_{t}(g(T-t))\right)^{\# g(T-t)}\left(\mathbf{W}_{0, t}^{T}\right) d t$ with

$$
\mathbf{W}_{0, t}^{T}: T_{x} M \longrightarrow T_{X_{t}^{T}(x)} M, \mathbf{W}_{0,0}^{T}=\mathrm{Id}_{T_{x} M} .
$$

By the over subscript $T$ we mean that the family of metrics is $g(T-t)$.

Proposition 2.3. Suppose that there exist $\bar{\alpha}, \underline{\alpha} \geq 0, \underline{K} \geq 0$ such that for all $t \in[0, T]:$

$$
\begin{gathered}
-\underline{\alpha} g(t) \leq \alpha(t) \leq \bar{\alpha} g(t), \\
-(d-1) \underline{K}^{2} g(t) \leq \operatorname{Ric}(t)
\end{gathered}
$$

then the $g(t)$-Brownian motion, and the $g(T-t)$-Brownian motion does not explode before the time $T$.

Proof. This is a sufficient condition but it is far from being necessary one, for the process to do not explode. Let $x, y \in M$ and let $d_{t}(x, y)$ be the Riemannian distance from $x$ to $y$ computed with the metric $g(t)$. Let $C u t_{t}(x)$ be the set of cutlocus of $x$ for the metric $g(t)$. Consider a fixed point $x_{0} \in M$, and $X_{t}$ a $g(t)$-Brownian motion starting at $X_{0}$. Using the Itô-Tanaka formula for $d_{t}\left(x_{0}, X_{t}\right)$ that have been proved for constant metric by Kendall, and generalized to $g(t)$-Brownian motion in [20] Theorem 2, we have:

$$
\begin{aligned}
& d_{t}\left(x_{0}, X_{t}\right) \\
& =d_{0}\left(x_{0}, X_{0}\right)+\int_{0}^{t} \mathbb{1}_{X_{s} \notin C u t_{s}\left(x_{0}\right)}\left(\frac{1}{2} \Delta_{g(s)} d_{s}\left(x_{0}, .\right)+\frac{\partial d_{s}\left(x_{0}, .\right)}{\partial s}\right)\left(X_{s}\right) d s \\
& +\int_{0}^{t} \mathbb{1}_{X_{s} \notin C u t_{s}\left(x_{0}\right)}\left\langle\nabla^{s} d_{s}\left(x_{0}, X_{s}\right), d^{\nabla_{s}} X_{s}\right\rangle_{g(s)}-L_{t},
\end{aligned}
$$


where $L_{t}$ is the local time at $C u t_{t}\left(x_{0}\right)$. The local time is non-decreasing non negative process that increase only when $X_{t}$ touches $C u t_{t}\left(x_{0}\right)$. Moreover the distance $d_{t}\left(x_{0}, x\right)$ is smooth if $x \notin C u t_{t}\left(x_{0}\right) \cup x_{0}$. Let $x \notin C u t_{t}\left(x_{0}\right)$ and $\gamma:\left[0, d_{t}\left(x_{0}, x\right)\right] \rightarrow M$ be the $g(t)$-geodesic from $x_{0}$ to $x$. We have :

$$
\frac{\partial d_{s}\left(x_{0}, x\right)}{\partial s}=\frac{1}{2} \int_{0}^{d_{s}\left(x_{0}, x\right)} \alpha(s)(\dot{\gamma}(u), \dot{\gamma}(u)) d u \leq \frac{\bar{\alpha}}{2} d_{s}\left(x_{0}, x\right) .
$$

Recall the Laplacian comparison Theorem:

$$
\Delta_{g(s)} d_{s}\left(x_{0}, x\right) \leq(d-1) \underline{K} \operatorname{coth}\left(\underline{K} d_{s}\left(x_{0}, x\right)\right) .
$$

We then get the following control of the drift term (using $x \operatorname{coth}(x) \leq 1+x$ for $x \geq 0)$, and $F(x):=(d-1)\left(\frac{\bar{\alpha} x}{(d-1)}+\frac{1}{x}+\underline{K}\right)$ :

$$
\begin{aligned}
& \left(\frac{1}{2} \Delta_{g(s)} d_{s}\left(x_{0}, .\right)+\frac{\partial d_{s}\left(x_{0}, .\right)}{\partial s}\right)(x) \\
& \leq \frac{1}{2}\left((d-1) \underline{K} \operatorname{coth}\left(\underline{K} d_{s}\left(x_{0}, x\right)+\bar{\alpha} d_{s}\left(x_{0}, x\right)\right)\right) \\
& \leq F\left(d_{s}\left(x_{0}, x\right)\right) .
\end{aligned}
$$

Since $\left\|\nabla^{g(t)} d_{t}\left(X_{0}, .\right)\right\|_{g(t)}=1$ and $C u t_{t}\left(x_{0}\right)$ have 0 as $g(t)$ volume, the martingale part of $d_{t}\left(x_{0}, X_{t}\right)$ is a real Brownian motion. We finish the proof using the comparison theorem of stochastic differential equation, and the usual criterion of non-explosion of a one dimensional diffusion. For the $g(T-t)$ Brownian motion, we simply to change $\bar{\alpha}$ by $\underline{\alpha}$ in the above formula.

Remark 2.4. For the backward Ricci flow, it is shown in [20] without any assumption as in the above proposition that the $g(t)$-Brownian motion does not explode. But the sufficient condition for the existence of the forward Ricci flow in complete Riemannian manifolds as given by Shi in [24, Theorem 1.1], that is the boundedness of the initial Riemannian tensor (for the metric $g(0)$ ) also gives a bound of the Ric tensor along the flow (for bounded time). Hence the conditions for non explosion of the $g(t)$-Brownian motion given in the above proposition is satisfied, at least for small time, if the initial metric satisfies Shi's condition for the complete manifolds.

In the following proposition $R_{t}^{T}$ is defined as $R_{t}$ but according to the family of metrics $g(T-t)$ instead of $g(t)$.

Proposition 2.5. Suppose that the $g(t)$-Brownian motion started at $x$ is non-explosive for the first point, and the $g(T-t)$-Brownian motion started at $x$ is non-explosive for the second point .

(1) If there exists $C \in \mathbb{R}$ such that $\operatorname{Ric}_{g(t)}-\alpha(t) \geq C g(t)$, then $R_{t}$ is a martingale, and for $\beta \geq 1$

$$
\mathbb{E}\left[R_{t}^{\beta}\right] \leq e^{\frac{1}{2} \beta(\beta-1) \frac{d_{0}^{2}(x, y)}{T^{2}} \frac{1-e^{-C t}}{C}} .
$$


(2) If there exists $\tilde{C} \in \mathbb{R}$ such that $\operatorname{Ric}_{g(t)}+\alpha(t) \geq \tilde{C} g(t)$ then $R_{t}^{T}$ is a martingale and for $\beta \geq 1$

$$
\mathbb{E}\left[\left(R_{t}^{T}\right)^{\beta}\right] \leq e^{\frac{1}{2} \beta(\beta-1) \frac{d_{T}^{2}(x, y)}{T^{2}} \frac{1-e^{-\tilde{C} t}}{\tilde{C}}} .
$$

If $C=0$ then we take for convention that for all $t, \frac{1-e^{-C t}}{C}=t$.

Proof. Without loss of generality, we just make the proof for $R_{t}$, the computation is the same as for $R_{t}^{T}$. Let $X_{t}(x)$ be a $g(t)$-Brownian motion and let $v \in T_{x} M$. We use short notation for the $g(t)$ parallel transport and the damped parallel transport along $X_{t}(x), /{ }_{t}:=/ /_{t}^{g(t), X .(x)}$ and $\mathbf{W}(X .(x))_{t}:=$ $\mathbf{W}_{t}^{g(t), X .(x)}$. Then we use the isometry property of the parallel transport, i.e., $/ / s:\left(T_{x} M, g(0)\right) \mapsto\left(T_{X_{s}(x)} M, g(s)\right)$, to deduce

$$
\begin{aligned}
& * d\left\langle\mathbf{W}(X .(x))_{s} v, \mathbf{W}(X .(x))_{s} v\right\rangle_{g(s)} \\
& =* d\left\langle/ /_{s}^{-1} \mathbf{W}(X .(x))_{s} v, / /{ }_{s}^{-1} \mathbf{W}(X .(x))_{s} v\right\rangle_{g(0)} \\
& =2\left\langle * d / /_{s}^{-1} \mathbf{W}(X .(x))_{s} v, / /{ }_{s}^{-1} \mathbf{W}(X .(x))_{s} v\right\rangle_{g(0)} \\
& =2\left\langle/ / s * d / /_{s}^{-1} \mathbf{W}(X .(x))_{s} v, \mathbf{W}(X .(x))_{s} v\right\rangle_{g(s)} \\
& =-\left\langle\left(\operatorname{Ric}_{g(s)}-\partial_{s}(g(s))\right)^{\# g(s)}\left(\mathbf{W}(X .(x))_{s} v\right), \mathbf{W}(X .(x))_{s} v\right\rangle_{g(s)} d s \\
& \leq-C\left\|\mathbf{W}(X .(x))_{s} v\right\|^{2} d s .
\end{aligned}
$$

By Gronwall's lemma we get

$$
\left\|\mathbf{W}(X .(x))_{s} v\right\|_{g(s)} \leq e^{-\frac{1}{2} C s}\|v\|_{g(0)} .
$$

Recall that $N_{t}:=-\frac{1}{T} \int_{0}^{t}\left\langle P_{0, \frac{s}{T}}^{g(s), X_{s}(.)} d^{\nabla_{s}} X_{s}(0), \mathbf{W}\left(X .\left(\frac{s}{T}\right)\right)_{s} \dot{\gamma}\left(\frac{s}{T}\right)\right\rangle_{g(s)}$, and $P_{0, \frac{s}{T}}^{g(s), X_{s}(.)}$ is a $g(s)$ isometry and $d^{\nabla_{s}} X_{s}(0)=/ /{ }_{s} e_{i} d w^{i}$ where $w$ is a $\mathbb{R}^{n}$ Brownian motion, and $\left(e_{i}\right)_{i=1 . . n}$ is an orthonormal basis of $T_{x} M$. Then

$$
\begin{aligned}
\langle N\rangle_{t} & =\frac{1}{T^{2}} \int_{0}^{t}\left\|\mathbf{W}\left(X .\left(\frac{s}{T}\right)\right)_{s} \dot{\gamma}\left(\frac{s}{T}\right)\right\|_{g(s)}^{2} d s \\
& \leq \frac{1}{T^{2}} \int_{0}^{t} e^{-C s}\left\|\dot{\gamma}\left(\frac{s}{T}\right)\right\|_{g(0)}^{2} d s \\
& \leq \frac{1}{T^{2}} d_{0}^{2}(x, y) \int_{0}^{t} e^{-C s} d s .
\end{aligned}
$$

So by Nokinov's criterion, $R_{t}$ is a martingale. Let $\beta \geq 1$,

$$
\begin{aligned}
\mathbb{E}\left[R_{t}^{\beta}\right] & =\mathbb{E}\left[e^{\beta N_{t}-\frac{\beta}{2}\langle N\rangle_{t}}\right] \\
& =\mathbb{E}\left[e^{\beta N_{t}-\frac{\beta^{2}}{2}\langle N\rangle_{t}} e^{\frac{\beta(\beta-1)}{2}\langle N\rangle_{t}}\right] \\
& \leq e^{\frac{1}{2} \beta(\beta-1) \frac{d_{0}^{2}(x, y)}{T^{2}} \frac{1-e^{-C t}}{C}} .
\end{aligned}
$$


By the same computation we have

$$
\begin{aligned}
\left\langle N^{T}\right\rangle_{t} & =\frac{1}{T^{2}} \int_{0}^{t}\left\|\mathbf{W}^{T}\left(X .\left(\frac{s}{T}\right)\right)_{s} \dot{\gamma}\left(\frac{s}{T}\right)\right\|_{g(T-s)}^{2} d s \\
& \leq \frac{1}{T^{2}} \int_{0}^{t} e^{-\tilde{C} s}\left\|\dot{\gamma}\left(\frac{s}{T}\right)\right\|_{g(T)}^{2} d s \\
& \leq \frac{1}{T^{2}} d_{T}^{2}(x, y) \int_{0}^{t} e^{-\tilde{C} s} d s .
\end{aligned}
$$

Thus $R_{t}^{T}$ is a martingale. Given $\beta \geq 1$ we have similarly (2.4).

Remark 2.6. In the case of Ricci flow, $\partial_{t} g(t)=-\operatorname{Ric}_{g(t)}$, then $\partial_{t} g(T-t)=$ Ric $_{g(T-t)}$ so the process $X_{t}^{T}(x)$ does not explode (we do not need proposition 2.3 , but [20]) and the condition of the above proposition is satisfied with $\tilde{C}=0$ and

$$
\mathbb{E}\left[\left(R_{T}^{T}\right)^{\beta}\right] \leq e^{\frac{1}{2} \beta(\beta-1) \frac{d_{T}^{2}(x, y)}{T}} .
$$

Using the horizontal $L_{t}$-diffusion, we could give a alternative proof of Theorem 3.2 in [4] (isoperimetric-type Harnack inequality) for the constant metric case, and also a generalisation for inhomogeneous diffusions.

Lemma 2.7. If there exists $\tilde{C} \in \mathbb{R}$ such that $\operatorname{Ric}_{g(t)}+\alpha(t) \geq \tilde{C} g(t)$ and if the $g(T-t)$-Brownian motion does not explode then for every measurable set A,

$$
P_{0, T}\left(\mathbb{1}_{A}\right)(x) \leq P_{0, T}\left(\mathbb{1}_{A_{0}^{\rho_{T}}}\right)(y) .
$$

Where $\rho_{T}:=e^{-\frac{\tilde{C} T}{2}} d_{T}(x, y)$ and $A_{0}^{\epsilon}:=\left\{z \in M\right.$ s.t. $\left.d_{0}(z, A) \leq \epsilon\right\}$

Proof. We could give a proof with the usual Kendall coupling, but we have to manage the different cutlocus. We prefer here give a proof using the horizontal $L_{t}$ diffusion in $C^{1}$ path space. Since the $g(T-t)$ - Brownian motion does not explode, it is the same for the $L_{T-t}$-horizontal diffusion. Let $\gamma$ be a $g(T)$ geodesic such that $\gamma(0)=x$ and $\gamma(1)=y$. By 2.1,

$$
\partial_{u} X_{t}(u)=\mathbf{W}^{T}\left(X_{.}^{T}(\gamma(u))\right)_{t}(\dot{\gamma}(u))
$$

and

$$
\left\|\mathbf{W}^{T}\left(X_{.}^{T}(x)\right)_{s} v\right\|_{g(T-s)} \leq e^{-\frac{1}{2} \tilde{C} s}\|v\|_{g(T)} .
$$

We then get

$$
d_{0}\left(X_{T}^{T}(x), X_{T}^{T}(y)\right) \leq \int_{0}^{1}\left\|\partial_{u} X_{t}(u)\right\|_{g(0)} d u \leq e^{-\frac{1}{2} \tilde{C} T} d_{T}(x, y)=\rho_{T} .
$$

Hence $\left\{X_{T}^{T}(x) \in A\right\} \subset\left\{X_{T}^{T}(y) \in A_{0}^{\rho_{T}}\right\}$ and

$$
P_{0, T}\left(\mathbb{1}_{A}\right)(x)=\mathbb{E}\left[\mathbb{1}_{A}\left(X_{T}^{T}(x)\right)\right] \leq \mathbb{E}\left[\mathbb{1}_{A_{0}^{\rho_{T}}}\left(X_{T}^{T}(y)\right)\right]=P_{0, T}\left(\mathbb{1}_{A_{0}^{\rho_{T}}}\right)(y) .
$$

Thus concludes the proof. 
Corollary 2.8. (1) If $\dot{g}=0$ and $\mathrm{Ric}_{g} \geq K$ then we can take $\rho_{T}=$ $e^{-\frac{K T}{2}} d(x, y)$. This as actually Theorem 3.2 in [4] for the Riemannian case.

(2) If $g(t)$ satisfies the Ricci flow, $\partial_{t} g(t)=-\operatorname{Ric}_{g(t)}$, so $X_{t}^{T}(x)$ does not explode ([20]) and since the damped parallel transport is an isometry we could take $\rho_{T}=d_{T}(x, y)$

We are now ready to give the Harnack inequality with power. Let $f$ be a solution of (1.1) and let $P_{0, T}$ be the inhomogeneous heat kernel associated to $(1.1)$, i.e.

$$
P_{0, T} f_{0}(x):=f(T, x)=\mathbb{E}_{x}\left[f_{0}\left(X_{T}^{T}\right)\right] .
$$

Theorem 2.9. Suppose that the $g(T-t)$-Brownian motion $X_{t}^{T}$ does not explode, and that the process $R_{t}^{T}$ is a martingale. Then for all $\alpha>1$ and $f_{0} \in C_{b}(M)$ we have :

$$
\left|P_{0, T} f_{0}\right|^{\alpha}(x) \leq \mathbb{E}\left[\left(R_{T}^{T}\right)^{\frac{\alpha}{\alpha-1}}\right]^{\alpha-1} P_{0, T}\left|f_{0}\right|^{\alpha}(y) .
$$

Moreover if there exists $\tilde{C} \in \mathbb{R}$ such that

$$
\operatorname{Ric}_{g(t)}+\alpha(t) \geq \tilde{C} g(t)
$$

then we have:

$$
\left|P_{0, T} f_{0}\right|^{\alpha}(x) \leq e^{\frac{\alpha}{2(\alpha-1)} \frac{d_{T}^{2}(x, y)}{T^{2}} \frac{1-e^{-\tilde{C} T}}{\tilde{C}}} P_{0, T}\left|f_{0}\right|^{\alpha}(y) .
$$

Proof. We write $\tilde{X}_{t}^{T}:=X_{t}^{T}\left(\frac{t}{T}\right)$ the diagonal process associated to the family of metrics $g(T-t)$, and use Proposition 2.2, and Hölder inequality:

$$
\begin{aligned}
\left|P_{0, T} f_{0}\right|^{\alpha}(x) & =\left|\mathbb{E}^{\mathbb{Q}}\left[f_{0}\left(\tilde{X}_{T}^{T}\right)\right]\right|^{\alpha} \\
& =\left|\mathbb{E}^{\mathbb{P}}\left[R_{T}^{T} f_{0}\left(\tilde{X}_{T}^{T}\right)\right]\right|^{\alpha} \\
& \leq \mathbb{E}^{\mathbb{P}}\left[\left(R_{T}^{T}\right)^{\alpha-1}\right]^{\alpha-1} \mathbb{E}^{\mathbb{P}}\left[\left|f_{0}\right|^{\alpha}\left(\tilde{X}_{T}^{T}\right)\right] \\
& =\mathbb{E}^{\mathbb{P}}\left[\left(R_{T}^{T}\right)^{\frac{\alpha}{\alpha-1}}\right]^{\alpha-1} \mathbb{E}_{y}^{\mathbb{P}}\left[\left|f_{0}\right|^{\alpha}\left(X_{T}^{T}(y)\right)\right] \\
& =\mathbb{E}^{\mathbb{P}}\left[\left(R_{T}^{T}\right)^{\frac{\alpha}{\alpha-1}}\right]^{\alpha-1} P_{0, T}\left|f_{0}\right|^{\alpha}(y) .
\end{aligned}
$$

The last part in the theorem is an application of Proposition 2.5.

We will denote by $\mu_{t}$ the volume measure associated to the metric $g(t)$, and for $A$ a measurable set, $\mu_{t}(A):=\int_{A} 1 d \mu_{t}$, and $B_{t}(x, r)$ the ball for the metric $g(t)$ of center $x$ and radius $r$.

Corollary 2.10. Suppose that the $g(T-t)$-Brownian motion $X_{t}^{T}$ does not explode, and there exists $\tilde{C} \in \mathbb{R}$ such that $\operatorname{Ric}_{g(t)}+\alpha(t) \geq \tilde{C} g(t)$. Moreover suppose that there exists a function $\tau:[0, T] \mapsto \mathbb{R}$ such that :

$$
\frac{1}{2} \operatorname{trace}_{g(t)}(\alpha(t))(y) \leq \tau(t), \quad \forall(t, y) \in[0, T] \times M
$$


then for $f_{0} \in L^{\alpha}\left(\mu_{0}\right)$

$$
\left|P_{0, T} f_{0}\right|(x) \leq \frac{e^{\frac{\int_{0}^{T} \tau(s) d s+1}{\alpha}}}{\left(\mu_{T}\left(B_{T}\left(x, \sqrt{\frac{2(\alpha-1) T^{2}}{\alpha\left(\frac{1-e^{-C} T}{\tilde{C}}\right)}}\right)\right)^{\frac{1}{\alpha}}\right.}\left\|f_{0}\right\|_{L^{\alpha}\left(\mu_{0}\right)} .
$$

Proof. By Proposition 2.5 $R_{t}^{T}$ is a martingale. If $f_{0} \in C_{b}(M) \cap L^{\alpha}\left(\mu_{0}\right)$ we apply Theorem 2.9 and get :

$$
\left|P_{0, T} f_{0}\right|^{\alpha}(x) \leq e^{\frac{\alpha}{2(\alpha-1)} \frac{d_{T}^{2}(x, y)}{T^{2}} \frac{1-e^{-\tilde{C} T}}{\tilde{C}}} P_{0, T}\left|f_{0}\right|^{\alpha}(y) .
$$

We integrate both sides along the ball $B_{T}\left(x, \sqrt{\frac{2(\alpha-1) T^{2}}{\alpha\left(\frac{1-e^{-C \tilde{C} T}}{\tilde{C}}\right)}}\right)$, with respect to the measure $\mu_{T}$, in $y$ and obtain :

$$
\begin{aligned}
& \mu_{T}\left(B_{T}\left(x, \sqrt{\frac{2(\alpha-1) T^{2}}{\alpha\left(\frac{1-e^{-\tilde{C} T}}{\tilde{C}}\right)}}\right)\right)\left|P_{0, T} f_{0}\right|^{\alpha}(x) \\
& \leq e \int_{B_{T}}\left(x, \sqrt{\frac{2(\alpha-1) T^{2}}{\alpha\left(\frac{1-e^{-\tilde{C} T}}{\tilde{C}}\right)}}\right) P_{0, T}\left|f_{0}\right|^{\alpha}(y) d \mu_{T}(y) \\
& \leq e \int_{M} P_{0, T}\left|f_{0}\right|^{\alpha}(y) d \mu_{T}(y) .
\end{aligned}
$$

We have that $\frac{d}{d t} \mu_{t}(y)=\frac{1}{2} \operatorname{trace}_{g(t)}(\alpha(t))(y) d \mu_{t}(y)$, and by the Stokes theorem we have :

$$
\begin{aligned}
\frac{d}{d t} \int_{M} P_{0, t}\left|f_{0}\right|^{\alpha}(y) d \mu_{t}(y) & =\int_{M} P_{0, t}\left|f_{0}\right|^{\alpha}(y) \frac{d}{d t} d \mu_{t}(y) \\
& \leq \tau(t) \int_{M} P_{0, t}\left|f_{0}\right|^{\alpha}(y) d \mu_{t}(y)
\end{aligned}
$$

We deduce that :

$$
\int_{M} P_{0, t}\left|f_{0}\right|^{\alpha}(y) d \mu_{t}(y) \leq e^{\int_{0}^{t} \tau(s) d s}\left\|f_{0}\right\|_{L^{\alpha}\left(\mu_{0}\right)}^{\alpha} .
$$

Hence for $f_{0} \in C_{b}(M) \cap L^{\alpha}\left(\mu_{0}\right)$

$$
\left|P_{0, T} f_{0}\right|(x) \leq \frac{e^{\frac{\int_{0}^{T} \tau(s) d s+1}{\alpha}}}{\left(\mu_{T}\left(B_{T}\left(x, \sqrt{\frac{2(\alpha-1) T^{2}}{\alpha\left(\frac{1-e^{-\tilde{C} T}}{\tilde{C}}\right)}}\right)\right)^{\frac{1}{\alpha}}\right.}\left\|f_{0}\right\|_{L^{\alpha}\left(\mu_{0}\right)} .
$$

We conclude by a classical density argument that the same inequality is true for $f_{0} \in L^{\alpha}\left(\mu_{0}\right)$.

Corollary 2.11. If the family of metric comes from the Ricci flow and if

$$
(\tau(t))=-\frac{1}{2} \inf _{y \in M} R(t, y)<\infty, \forall t \in[0, T]
$$


where $R(t, y)$ is the scalar curvature at $y$ for the metric $g(t)$ then we have

$$
\left|P_{0, T} f_{0}\right|(x) \leq \frac{e^{\frac{\int_{0}^{T} \tau(s) d s+1}{\alpha}}}{\left(\mu_{T}\left(B_{T}\left(x, \sqrt{\frac{2(\alpha-1) T}{\alpha}}\right)\right)\right)^{\frac{1}{\alpha}}}\left\|f_{0}\right\|_{L^{\alpha}\left(\mu_{0}\right)} .
$$

If $\inf _{x \in M}\left(\mu_{T}\left(B_{T}\left(x, \sqrt{\frac{2(\alpha-1) T}{\alpha}}\right)\right)\right)=: C_{T}>0$ then as a linear operator:

$$
\left\|P_{0, T}\right\|_{L^{\alpha}\left(\mu_{0}\right) \mapsto L^{\infty}\left(\mu_{0}\right)} \leq \frac{e^{\frac{\int_{0}^{T} \tau(s) d s+1}{\alpha}}}{C_{T}^{\frac{1}{\alpha}}} .
$$

Proof. If $g(t)$ comes from Ricci flow then $g(T-t)$ satisfies a backward Ricci flow. Then the process $X_{t}^{T}(x)$ does not explode before $T$ [20]. Moreover we have $\tilde{C}=0$ in Proposition 2.5, then the process $R_{t}^{T}$ is a martingale and we could apply the above corollary.

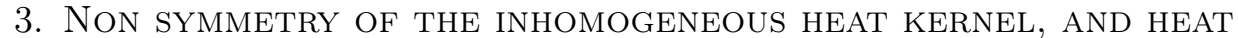 KERNEL ESTIMATE}

Unfortunately the non homogeneous heat kernel is non symmetric in general. The goal of this section is to by-pass this difficulty. This will be achieved by the study of the dual process and time reverse.

Let $\frac{\partial}{\partial t} g(t):=\alpha(t)$ where $\alpha$ is a time-dependent symmetric 2-tensor. We suppose that there exist functions $\tau(t)$ and $\underline{\tau}(t)$ such that :

$$
\left\{\begin{array}{l}
\frac{1}{2} \sup _{y \in M} \operatorname{trace}_{g(t)}(\alpha(t))(y) \leq \tau(t) \\
\frac{1}{2} \inf _{y \in M} \operatorname{trace}_{g(t)}(\alpha(t))(y) \geq \underline{\tau}(t) .
\end{array}\right.
$$

Consider the following heat operator where the subscript mean the variable in which we differentiate: $L_{t, x}:=-\frac{\partial}{\partial t}+\frac{1}{2} \Delta_{g(t)}$. Let $x, y \in M$ and $0<\tau<$ $\sigma \leq t$. Denote by $P(x, t, y, \tau)$ the fundamental solution of

$$
\left\{\begin{array}{l}
L_{t, x} P(x, t, y, \tau)=0 \\
\lim _{t \searrow \tau} P(., t, y, \tau)=\delta_{y}(.)
\end{array}\right.
$$

Using Itô's formula we obtain as in [10]:

$$
X_{t-\tau}^{t-\dot{\tau}}(x) \stackrel{\mathcal{L}}{=} P(x, t, y, \tau) d \mu_{\tau}(y)
$$

Let $v, u \in C^{1,2}(\mathbb{R}, M)$, the space of functions that are differentiable in time, and differentiable twice in space. Consider the adjoint operator $L^{*}$ of $L$ with respect to $\langle L u, v\rangle:=\int_{0}^{T} \int_{M}(L u) v d \mu_{t} d t$. As in Guenther [17], it satisfies

$$
L_{t, x}^{*}=\frac{1}{2} \Delta_{t}+\frac{\partial}{\partial_{t}}+\frac{1}{2} \operatorname{trace}_{g(t)}(\alpha(t)) .
$$

The fundamental solution $P^{*}(y, \tau, x, t)$ of $L^{*}$, satisfies : 


$$
\left\{\begin{array}{l}
L_{\tau, y}^{*} P^{*}(y, \tau, x, t)=0 \\
\lim _{\tau \nearrow t} P^{*}(., \tau, x, t)=\delta_{x}(.)
\end{array}\right.
$$

Using Duhamel's principle the adjoint property yields:

$$
P(x, t, y, \tau)=P^{*}(y, \tau, x, t) .
$$

After a time reversal, $P^{*}(y, t-s, x, t)$ satisfies the following heat equation :

$$
\left\{\begin{array}{l}
\partial_{s} P^{*}(y, t-s, x, t)=\frac{1}{2} \Delta_{g(t-s), y} P^{*}+\frac{1}{2} \operatorname{trace}_{g(t-s)}(\alpha(t-s))(y) P^{*} \\
\lim _{s \searrow 0} P^{*}(y, t-s, x, t)=\delta_{y} .
\end{array}\right.
$$

Using the Feynman-Kac formula, we conclude that :

$$
P^{*}(y, t-s, x, t) \leq e^{\frac{1}{2} \int_{0}^{s} \tau(t-u) d u} \bar{P}(y, s, x, t),
$$

where $\bar{P}(y, s, x, t)$ be the fundamental solution of

$$
\left\{\begin{array}{l}
\partial_{s} f(s, x)=\frac{1}{2} \Delta_{g(t-s)} f(s, x) \\
f(0, x)=f_{0}(x)
\end{array}\right.
$$

i.e., $\bar{P}(y, s, x, t)$ satisfies :

$$
\left\{\begin{array}{l}
\partial_{s} \bar{P}(y, s, x, t)=\frac{1}{2} \Delta_{g(t-s), y} \bar{P}(y, s, x, t) \\
\lim _{s \searrow_{0}} \bar{P}(., s, x, t)=\delta_{x}(.) .
\end{array}\right.
$$

Theorem 3.1. Suppose that (3.1) is satisfied and that:

- the $g(s)$-Brownian motion does not explode before the time $\frac{t}{2}$ and there exists $C \in \mathbb{R}$ such that $\forall s \in\left[0, \frac{t}{2}\right]$ :

$$
\operatorname{Ric}_{g(s)}-\alpha(s) \geq C g(s)
$$

- the $g(t-s)$-Brownian motion does not explode before the time $\frac{t}{2}$ and there exist $\tilde{C} \in \mathbb{R}$ such that $\forall s \in\left[0, \frac{t}{2}\right]$ :

$$
\operatorname{Ric}_{g(t-s)}+\alpha(t-s) \geq \tilde{C} g(t-s) .
$$

Then the fundamental solution of (1.1) that we note $P(x, t, y, 0)$ satisfies for all $0<t<T_{c}$ :

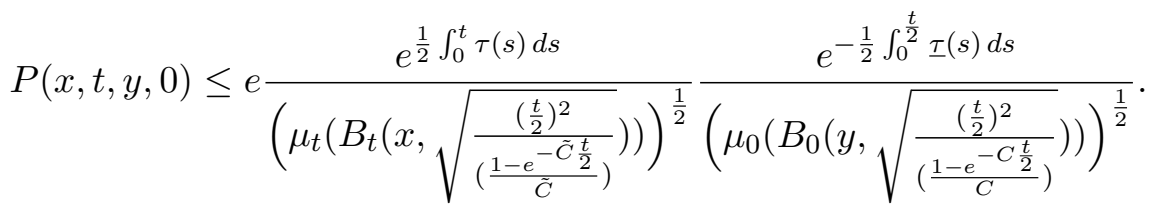


Proof. By the Chapman-Kolmogorov formula we have:

$$
\begin{aligned}
P(x, t, y, 0) & =\int_{M} P\left(x, t, z, \frac{t}{2}\right) P\left(z, \frac{t}{2}, y, 0\right) d \mu_{\frac{t}{2}}(z) \\
& =\int_{M} P\left(x, t, z, \frac{t}{2}\right) P^{*}\left(y, 0, z, \frac{t}{2}\right) d \mu_{\frac{t}{2}}(z) \\
& \leq\left(\int_{M}\left(P\left(x, t, z, \frac{t}{2}\right)\right)^{2} d \mu_{\frac{t}{2}}(z)\right)^{\frac{1}{2}}\left(\int_{M}\left(P^{*}\left(y, 0, z, \frac{t}{2}\right)\right)^{2} d \mu_{\frac{t}{2}}(z)\right)^{\frac{1}{2}} .
\end{aligned}
$$

Recall that $P\left(x, \frac{t}{2}+s, z, \frac{t}{2}\right)$ is the fundamental solution, which starts at $\delta_{x}$ at time $s=0$, of :

$$
\left\{\begin{array}{l}
\partial_{s} f(s, x)=\frac{1}{2} \Delta_{g\left(\frac{t}{2}+s\right)} f(s, x) \\
f(0, x)=f_{0}(x)
\end{array}\right.
$$

Then we have :

$$
P_{0, \frac{t}{2}} f_{0}(x):=f\left(\frac{t}{2}, x\right)=\mathbb{E}\left[f_{0}\left(X_{\frac{t}{2}}^{t-\cdot}(x)\right)\right] .
$$

According to the proof of Corollary 2.10, for $f_{0} \in C_{b}(M) \cap L^{2}\left(\mu_{\frac{t}{2}}\right)$ :

$$
\left|P_{0, \frac{t}{2}} f_{0}\right|(x) \leq \frac{e^{\frac{\int_{0}^{\frac{t}{2}} \tau\left(\frac{t}{2}+s\right) d s+1}{2}}}{\left(\mu _ { t } \left(B _ { t } \left(x, \sqrt{\left.\left.\frac{\left(\frac{t}{2}\right)^{2}}{\left(\frac{1-e^{-\tilde{C}\left(\frac{t}{2}\right)}}{\tilde{C}}\right)}\right)\right)^{\frac{1}{2}}}\right.\right.\right.}\left\|f_{0}\right\|_{L^{2}\left(\mu_{\frac{t}{2}}\right)} .
$$

Given $x_{0} \in M$ and $n \in \mathbb{N}$, we apply the above inequality to $f_{0}(y):=$ $P\left(x, t, y, \frac{t}{2}\right) \wedge\left(n \mathbb{1}_{B\left(x_{0}, n\right)}(y)\right)$ to obtain :

$$
\begin{aligned}
& \int_{M}\left(P\left(x, t, z, \frac{t}{2}\right) \wedge\left(n \mathbb{1}_{B\left(x_{0}, n\right)}(z)\right)\right)^{2} d \mu_{\frac{t}{2}}(z) \\
& \leq \int_{M} P\left(x, t, z, \frac{t}{2}\right)\left(P\left(x, t, z, \frac{t}{2}\right) \wedge n \mathbb{1}_{B\left(x_{0}, n\right)}(z)\right) d \mu_{\frac{t}{2}}(z) \\
& \leq \frac{e^{\frac{\int_{0}^{\frac{t}{2}} \tau\left(\frac{t}{2}+s\right) d s+1}{2}}}{\left(\mu _ { t } \left(B _ { t } \left(x, \sqrt{\left.\left.\frac{\left(\frac{t}{2}\right)^{2}}{\left(\frac{1-e^{-\tilde{C}\left(\frac{t}{2}\right)}}{\tilde{C}}\right)}\right)\right)^{\frac{1}{2}}}\left(\int_{M}\left(P\left(x, t, z, \frac{t}{2}\right) \wedge\left(n \mathbb{1}_{B\left(x_{0}, n\right)}(z)\right)\right)^{2} d \mu_{\frac{t}{2}}(z)\right)^{\frac{1}{2}} .\right.\right.\right.}
\end{aligned}
$$

Letting $n$ goes to infinity, we obtain that $z \rightarrow P\left(x, t, z, \frac{t}{2}\right)$ is in $L^{2}\left(\mu_{\frac{t}{2}}\right)$ for $t>0$, and that:

$$
\left(\int_{M}\left(P\left(x, t, z, \frac{t}{2}\right)\right)^{2} d \mu_{\frac{t}{2}}(z)\right)^{\frac{1}{2}} \leq \frac{e^{\frac{\int_{0}^{\frac{t}{2}} \tau\left(\frac{t}{2}+s\right) d s+1}{2}}}{\left(\mu _ { t } \left(B _ { t } \left(x, \sqrt{\left.\left.\frac{\left(\frac{t}{2}\right)^{2}}{\left(\frac{1-e^{-\tilde{C}\left(\frac{t}{2}\right)}}{\tilde{C}}\right)}\right)\right)^{\frac{1}{2}}}\right.\right.\right.} .
$$


Recall that:

$$
P^{*}\left(y, 0, x, \frac{t}{2}\right) \leq e^{\frac{1}{2} \int_{0}^{\frac{t}{2}} \tau(u) d u} \bar{P}\left(y, \frac{t}{2}, x, \frac{t}{2}\right),
$$

where $\bar{P}\left(y, \frac{t}{2}, x, \frac{t}{2}\right)$ is the heat kernel at time $\frac{t}{2}$, which starts at time 0 at $\delta_{y}$, of the following equation:

$$
\left\{\begin{array}{l}
\partial_{s} f(s, x)=\frac{1}{2} \Delta_{g\left(\frac{t}{2}-s\right)} f(s, x) \\
f(0, x)=f_{0}(x)
\end{array}\right.
$$

We also have:

$$
\bar{P}_{0, \frac{t}{2}} f_{0}(x):=f\left(\frac{t}{2}, x\right)=\mathbb{E}\left[f_{0}\left(X_{\frac{t}{2}}^{g(\cdot)}(x)\right)\right]
$$

To make a direct link with 2.10, we could think that the family of metrics is $s \mapsto g\left(\frac{t}{2}-s\right)$, so many changes of signs are involved. However, the proof of the following is the same as the one of Corollary 2.10. We get for $f_{0} \in$ $B_{b}(M) \cap L^{2}\left(\mu_{\frac{t}{2}}\right):$

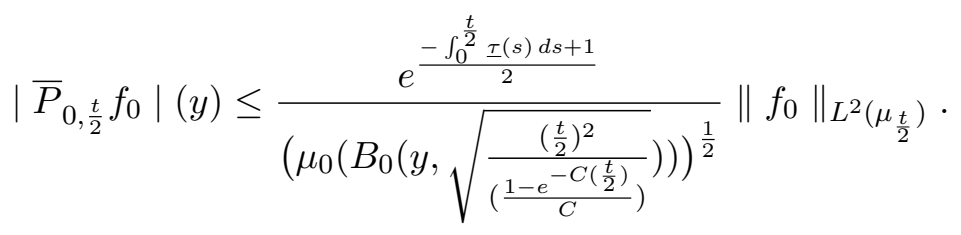

Similarly $z \rightarrow \bar{P}\left(y, \frac{t}{2}, z, \frac{t}{2}\right)$ is in $L^{2}\left(\mu_{\frac{t}{2}}\right)$ and

$$
\left(\int_{M}\left(\bar{P}\left(y, \frac{t}{2}, z, \frac{t}{2}\right)\right)^{2} d \mu_{\frac{t}{2}}(z)\right)^{\frac{1}{2}} \leq \frac{e^{\frac{-\int_{0}^{\frac{t}{2}} \frac{\tau(s) d s+1}{2}}{2}}}{\left(\mu _ { 0 } \left(B_{0}\left(y, \sqrt{\left.\frac{\left(\frac{t}{2}\right)^{2}}{\left(\frac{1-e^{-C\left(\frac{t}{2}\right)}}{C}\right)}\right)}\right)^{\frac{1}{2}}\right.\right.}
$$

We obtain :

$$
\begin{aligned}
& \left(\int_{M}\left(P^{*}\left(y, 0, z, \frac{t}{2}\right)\right)^{2} d \mu_{\frac{t}{2}}(z)\right)^{\frac{1}{2}} \\
& \leq e^{\frac{1}{2} \int_{0}^{\frac{t}{2}} \tau(u) d u}\left(\int_{M} \bar{P}^{2}\left(y, \frac{t}{2}, z, \frac{t}{2}\right) d \mu_{\frac{t}{2}}(z)\right)^{\frac{1}{2}} \\
& \leq e^{\frac{1}{2} \int_{0}^{\frac{t}{2}} \tau(u) d u} \frac{e^{\frac{-\int_{0}^{\frac{t}{2}} \frac{\tau}{2}(s) d s+1}{2}}}{\left(\mu _ { 0 } \left(B _ { 0 } \left(y, \sqrt{\left.\left.\frac{\left(\frac{t}{2}\right)^{2}}{\left(\frac{1-e^{-C\left(\frac{t}{2}\right)}}{C}\right)}\right)\right)^{\frac{1}{2}}} .\right.\right.\right.}
\end{aligned}
$$

Remark 3.2. Having a heat kernel estimate for the heat equation we have simultaneously a kernel estimate of conjugate equation. 
Remark 3.3. The hypothesis $\operatorname{Ric}_{g(t-s)}+\alpha(t-s) \geq \tilde{C} g(t-s)$, for $s \in\left[0, \frac{t}{2}\right]$ is a kind of quantitative super Ricci flow as defined in [22] (if $\tilde{C}=0$ this is exactly the definition of super Ricci flow). This quantitative version of super Ricci flow allow us to control the rate of expansion of the damped parallel transport along the $g(t-$.)-Brownian motion.

The hypothesis $\operatorname{Ric}_{g(s)}-\alpha(s) \geq C g(s)$ for $s \in\left[0, \frac{t}{2}\right]$ allow us to control the rate of expansion of the damped parallel transport along the dual process, namely the process associated to $\bar{P}$ i.e. $g($.)-Brownian motion.

Remark 3.4. If $g(t)=g(0)$ is constant, and $\operatorname{Ric}_{g(0)} \geq 0$ we have $\tau(t)=$ $\underline{\tau}(t)=0, C=\tilde{C}=0$ and we deduce a Li-Yau on-diagonal estimate of the usual heat equation on complete manifolds as in [21] (up to some constant):

$$
P_{t}(x, y) \leq e \frac{1}{\left(\mu_{0}\left(B_{0}\left(x, \sqrt{\frac{t}{2}}\right)\right)\right)^{\frac{1}{2}}} \frac{1}{\left(\mu_{0}\left(B_{0}\left(y, \sqrt{\frac{t}{2}}\right)\right)^{\frac{1}{2}}\right.} .
$$

Using the symmetry of the heat kernel in the constant metric case we do not need to consider the dual as in the above theorem.

\section{GRigor'yan tricks, on-Diagonal estimate to Gaussian ESTIMATE, THE RiCCI FLOW CASE}

In this section we use the on-diagonal estimate of the previous section to derive a Gaussian type estimate of the minimal heat kernel coupled with Ricci flow (for complete manifold with non negative Ricci curvature). The proof involves several steps. In particular, we use a modification of Grigor'yan tricks $[16,15]$ to control integrability of the square of the heat kernel outside some ball, combined to an adapted version of Hamilton entropy estimate to control the difference of the heat kernel at two points. This type of strategy, is a modification of differents arguments which appears in the literature on the Ricci flow (e.g. Cao-Zhang [5]).

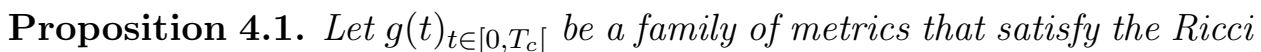
flow i.e. $\dot{g}(t)=-\operatorname{Ric}_{g(t)}$. Suppose that for all $t \in\left[0, T_{c}\left[, \operatorname{Ric}_{g(t)} \geq 0\right.\right.$ then we have the following on-diagonal estimate for the heat kernel and so for the conjugate heat kernel:

$$
P(x, t, y, 0) \leq e \frac{e^{-\frac{1}{4} \int_{\frac{t}{2}}^{t} \inf _{M} R(s, .) d s}}{\left(\mu_{t}\left(B_{t}\left(x, \sqrt{\frac{t}{2}}\right)\right)^{\frac{1}{2}}\right.} \frac{e^{\frac{1}{4} \int_{0}^{\frac{t}{2}}\left(\sup _{M} R(s, .)-\inf _{M} R(s, .)\right) d s}}{\left(\mu_{0}\left(B_{0}\left(y, \sqrt{\frac{t}{2}}\right)\right)\right)^{\frac{1}{2}}} .
$$

Proof. We could use Proposition 2.3 with $\bar{K}=0$ and $\bar{\alpha}=0$ to get the nonexplosion of the $g(t)$-Brownian motion, and as in [20] the $g(T-t)$-Brownian motion does not explode. We then use Theorem 3.1 with $C=0$ and $\tilde{C}=0$ 
to get the following on-diagonal estimate, for all $t \in] 0, T_{c}[$ :

$$
P(x, t, y, 0) \leq e \frac{e^{\frac{1}{2} \int_{0}^{t} \tau(s) d s}}{\left(\mu_{t}\left(B_{t}\left(x, \sqrt{\frac{t}{2}}\right)\right)\right)^{\frac{1}{2}}} \frac{e^{-\frac{1}{2} \int_{0}^{\frac{t}{2}} \underline{\tau}(s) d s}}{\left(\mu_{0}\left(B_{0}\left(y, \sqrt{\frac{t}{2}}\right)\right)\right)^{\frac{1}{2}}} .
$$

Recall that in the case of Ricci flow : $\tau(s)=-\frac{1}{2} \inf _{M} R(s,) \leq$.0 .

Remark 4.2. Note that in the proof of Theorem 3.1 the time $\frac{t}{2}$ is arbitrary and so in Proposition 4.1. Thus if we have only a control of $\sup _{M} R$ for small time, we also have a on-diagonal estimate.

We start this section by the following Hamilton estimate.

Lemma 4.3. Let $f$ be a positive solution of $(1.1)$, where $\alpha_{i, j}(t)=-\left(\operatorname{Ric}_{g(t)}\right)_{i, j}$, $t \in] 0, T_{c}\left[\right.$ and $M_{\frac{t}{2}}:=\sup _{x \in M} f\left(\frac{t}{2}, x\right)$ then for all $x, y \in M$,

$$
f(t, x) \leq \sqrt{f(t, y)} \sqrt{M_{\frac{t}{2}}} e^{\frac{d_{t}^{2}(x, y)}{t}} .
$$

Proof. By the homogeneity of the desired inequality under multiplication by a constant, and the linearity of the heat equation, we can suppose that $f>1$, by taking for $\epsilon>0, f_{\epsilon}=\frac{f+2 \epsilon}{\inf _{M} f+\epsilon}$ and take the limit in $\epsilon$.

Since no confusion could arise, we will simply write without subscript $\nabla,\|\cdot\|$, and $\|\cdot\|_{H S}$, for $\nabla^{g(t)},\|\cdot\|_{g(t)}, \ldots$ Recall the Hilbert-Schmidt norm is defined as $\|\alpha\|_{H S}^{2}=g^{i l} g^{j m} \alpha_{i j} \alpha_{l m}$, for a 2-tensor $\alpha:=\alpha_{i j} d x_{i} \otimes d x_{j}$.

Using an orthonormal frame and Weitzenbock's formula, we obtain the following equation

$$
\begin{aligned}
\left(-\partial_{t}+\right. & \left.\frac{1}{2} \Delta_{g(t)}\right)\left(\frac{\|\nabla f\|^{2}}{f}\right)(t, x)= \\
& \frac{1}{f}\left(\left\|\operatorname{Hess} f-\frac{\nabla f \otimes \nabla f}{f}\right\|_{H S}^{2}+\left(\operatorname{Ric}_{g(t)}+\dot{g}\right)(\nabla f, \nabla f)\right)(t, x) .
\end{aligned}
$$

Thus, in the case of Ricci flow

$$
\left(-\partial_{t}+\frac{1}{2} \Delta_{g(t)}\right)\left(\frac{\|\nabla f\|^{2}}{f}\right) \geq 0
$$

By a direct computation

Let

$$
\left(-\partial_{t}+\frac{1}{2} \Delta_{g(t)}\right)(f \log f)(t, x)=\frac{1}{2} \frac{\|\nabla f\|^{2}}{f}(t, x) .
$$

$$
N_{s}:=h(s) \frac{\|\nabla f\|^{2}}{f}\left(t-s, X_{s}^{t}(x)\right)+(f \log f)\left(t-s, X_{s}^{t}(x)\right),
$$

where $X_{s}^{t}(x)$ is a $g(t-s)$-Brownian motion started at $x$. If $h(s):=\frac{t / 2-s}{2}$ then by Itô formula, it is easy to see that $N_{s}$ is a super-martingale. So we have :

$$
\mathbb{E}\left[N_{0}\right] \leq \mathbb{E}\left[N_{\frac{t}{2}}\right],
$$


that is :

$$
\begin{aligned}
\frac{t}{4} \frac{\|\nabla f\|^{2}}{f}(t, x)+(f \log f)(t, x) & \leq \mathbb{E}\left[(f \log f)\left(\frac{t}{2}, X_{\frac{t}{2}}^{t}(x)\right)\right] \\
& \leq \mathbb{E}\left[f\left(\frac{t}{2}, X_{\frac{t}{2}}^{t}(x)\right)\right] \log \left(M_{\frac{t}{2}}\right) \\
& =f(t, x) \log \left(M_{\frac{t}{2}}\right)
\end{aligned}
$$

where we use $f>1$ and that $f\left(t-s, X_{s}^{t}(x)\right)$ is a martingale. The above computation yields

$$
\frac{\|\nabla f\|}{f}(t, x) \leq \frac{2}{\sqrt{t}} \sqrt{\log \left(\frac{M_{\frac{t}{2}}}{f(t, x)}\right)}
$$

and consequently

$$
\left\|\nabla \sqrt{\log \left(\frac{M_{\frac{t}{2}}}{f(x, t)}\right)}\right\| \leq \frac{1}{\sqrt{t}} .
$$

After integrating this inequality along a $g(t)$-geodesic between $x$ and $y$, we get

that yields to

$$
\sqrt{\log \left(\frac{M_{\frac{t}{2}}}{f(y, t)}\right)} \leq \sqrt{\log \left(\frac{M_{\frac{t}{2}}}{f(x, t)}\right)}+\frac{d_{t}(x, y)}{\sqrt{t}}
$$

$$
f(t, x) \leq \sqrt{f(t, y)} \sqrt{M_{\frac{t}{2}}} e^{\frac{d_{t}^{2}(x, y)}{t}} .
$$

Now, we adapt the argument of Grigor'yan to the situation of Ricci flow (with non negative Ricci curvature).

Lemma 4.4. Suppose that (3.1) is satisfied, the family of metrics $g(t)$ comes from the Ricci flow, and let $B$ be a measurable set, then:

$$
\frac{e^{-\frac{1}{2} \int_{0}^{t} \tau(s) d s}}{\mu_{0}(B)^{\frac{1}{2}}} \leq \frac{1}{\mu_{t}(B)^{\frac{1}{2}}} \leq \frac{e^{-\frac{1}{2} \int_{0}^{t} \underline{\tau}(s) d s}}{\mu_{0}(B)^{\frac{1}{2}}} .
$$

Moreover if $\operatorname{Ric}_{g(t)} \geq 0$ for all $t \in\left[0, T_{c}[\right.$ then for all $x \in M$ and $r>0$ we have :

$$
\frac{1}{\mu_{0}\left(B_{t}(x, r)\right)^{\frac{1}{2}}} \leq \frac{1}{\mu_{0}\left(B_{0}(x, r)\right)^{\frac{1}{2}}} .
$$

Proof. Recall that:

$$
\frac{d}{d t} \mu_{t}=\frac{1}{2} \operatorname{trace}_{g(t)}(\dot{g}(t)) \mu_{t} .
$$

In the case of a Ricci flow this becomes $\frac{d}{d t} \mu_{t}(d x)=-\frac{1}{2} R(x, t) \mu_{t}(d x)$. Thus, the first inequality of the lemma follows from an integration. For the second point, it is clear that Ric $\geq 0$ yields that $d_{t}(x, y)$ is non increasing in time. Then $B_{0}(x, r) \subset B_{t}(x, r)$, which clearly gives $\frac{1}{\mu_{0}\left(B_{t}(x, r)\right)^{\frac{1}{2}}} \leq \frac{1}{\mu_{0}\left(B_{0}(x, r)\right)^{\frac{1}{2}}}$. 
The above lemma immediately yields the following remark.

Remark 4.5. Suppose $\dot{g}(t)=-\operatorname{Ric}_{g(t)}$ and $\operatorname{Ric}_{g(.)} \geq 0$. Using the estimate in Lemma 4.1 and Lemma 4.4 we have the following estimate :

$$
P(x, t, y, 0) \leq e \frac{e^{\frac{1}{4} \int_{0}^{t}\left(\sup _{M} R(s, .)-\inf _{M} R(s, .)\right) d s}}{\left(\mu_{0}\left(B_{0}\left(x, \sqrt{\frac{t}{2}}\right)\right)\right)^{\frac{1}{2}}} \frac{e^{\frac{1}{4} \int_{0}^{\frac{t}{2}} \sup _{M} R(s, .) d s}}{\left(\mu_{0}\left(B_{0}\left(y, \sqrt{\frac{t}{2}}\right)\right)\right)^{\frac{1}{2}}} .
$$

Proposition 4.6. Let $g(t)$ be a solution of Ricci flow such that $\operatorname{Ric}_{g}(t) \geq 0$, and let $r>0, t_{0}>t \geq 0$, and define:

$$
\xi(y, t)=\left\{\begin{array}{lll}
\frac{-\left(r-d_{t}(x, y)\right)^{2}}{\left(t_{0}-t\right)} & \text { if } & d_{t}(x, y) \leq r \\
0 & \text { if } & d_{t}(x, y) \geq r
\end{array}\right.
$$

and $\Lambda(t)=\frac{1}{2} \int_{0}^{t} \inf _{x \in M}(R(s, x)) d s$. If $f$ is a solution of (1.1) then for $t_{2}<t_{1}<t_{0}$ :

$$
\int_{M} f^{2}\left(t_{1}, y\right) e^{\xi\left(y, t_{1}\right)} \mu_{t_{1}}(d y) \leq e^{-\left(\Lambda\left(t_{1}\right)-\Lambda\left(t_{2}\right)\right)} \int_{M} f^{2}\left(t_{2}, y\right) e^{\xi\left(y, t_{2}\right)} \mu_{t_{2}}(d y) .
$$

Proof. Let $\gamma(s)$ be the $g(t)$-geodesic between $x$ and $y$. Using Remark 6 in [22] and the fact that $\operatorname{Ric}_{g(t)} \geq 0$ we get

$$
\begin{aligned}
\frac{d}{d t} d_{t}^{2}(x, y) & =\frac{d}{d t} \int_{0}^{1}\|\dot{\gamma}(s)\|_{g(t)}^{2} d s \\
& =-\int_{0}^{1} \operatorname{Ric}_{g(t)}(\dot{\gamma}(s), \dot{\gamma}(s)) d s \leq 0 .
\end{aligned}
$$

So for $y \in B_{t}(x, r)$ we have

$$
\frac{d}{d t} \xi(y, t) \leq \frac{-\left(r-d_{t}(x, y)\right)^{2}}{\left(t_{0}-t\right)^{2}}
$$

We also have

$$
\left\|\nabla^{t} \xi(y, t)\right\|_{g(t)}^{2}=\frac{4\left(r-d_{t}(x, y)\right)^{2}}{\left(t_{0}-t\right)^{2}}
$$

Then

$$
\frac{d}{d t} \xi(y, t) \leq-\frac{\left\|\nabla^{t} \xi(y, t)\right\|_{g(t)}^{2}}{4}
$$


and the above inequality is clear if $y \notin B_{t}(x, r)$. Let $f(t, x)$ a solution of (1.1) then we have :

$$
\begin{aligned}
& \frac{d}{d t} \int_{M} f^{2}(t, y) e^{\xi(y, t)} \mu_{t}(d y) \\
& =\int_{M}\left(f(t, y) \Delta_{t} f(t, y)+f^{2}(t, y) \frac{d}{d t} \xi(y, t)-\frac{R(y, t)}{2} f^{2}(t, y)\right) e^{\xi(y, t)} \mu_{t}(d y) \\
& =\int_{M}-\left\langle\nabla^{t} f, \nabla^{t}\left(f e^{\xi}\right)\right\rangle_{g(t)}+f^{2} \frac{d}{d t} \xi e^{\xi}-\frac{R}{2} f^{2} e^{\xi} \mu_{t}(d y) \\
& =\int_{M}\left(-\left\langle\nabla^{t} f, \nabla^{t} f\right\rangle_{g(t)}-\left\langle\nabla^{t} f, f \nabla^{t} \xi\right\rangle_{g(t)}+f^{2} \frac{d}{d t} \xi\right) e^{\xi}-\frac{R}{2} f^{2} e^{\xi} \mu_{t}(d y) \\
& \leq-\int_{M}\left(\left\langle\nabla^{t} f, \nabla^{t} f\right\rangle+2\left\langle\nabla^{t} f, f \nabla^{t} \frac{\xi}{2}\right\rangle+f^{2} \frac{\left\|\nabla^{t} \xi\right\|^{2}}{4}\right) e^{\xi}-\frac{R}{2} f^{2} e^{\xi} \mu_{t}(d y) \\
& =-\int_{M}\left(\left\langle\nabla^{t} f, \nabla^{t} f\right\rangle+2\left\langle\nabla^{t} f, f \nabla^{t} \frac{\xi}{2}\right\rangle+f^{2} \frac{\left\|\nabla^{t} \xi\right\|^{2}}{4}\right) e^{\xi}-\frac{R}{2} f^{2} e^{\xi} \mu_{t}(d y) \\
& =-\int_{M}\left\|\nabla^{t} f+f \nabla^{t} \frac{\xi}{2}\right\|_{g(t)}^{2} \mu_{t}(d y)-\int_{M} \frac{R}{2} f^{2} e^{\xi} \mu_{t}(d y) \\
& \leq-\int_{M} \frac{R}{2} f^{2} e^{\xi} \mu_{t}(d y) .
\end{aligned}
$$

The result follows.

We define

$$
I_{r}(t):=\int_{M \backslash B_{t}(x, r)} f^{2}(t, y) \mu_{t}(d y) .
$$

Proposition 4.7. Under the same assumptions as in Proposition 4.6. Let $\rho<r$ and $f$ be a solution of (1.1). We have:

$$
I_{r}\left(t_{1}\right) \leq e^{-\left(\Lambda\left(t_{1}\right)-\Lambda\left(t_{2}\right)\right)}\left(I_{\rho}\left(t_{2}\right)+e^{\frac{-(r-\rho)^{2}}{\left(t_{1}-t_{2}\right)}} \int_{M} f^{2}\left(t_{2}, y\right) \mu_{t_{2}}(d y)\right)
$$

for $t_{2}<t_{1}$. 
Proof. For $t_{2}<t_{1}$

$$
\begin{aligned}
I_{r}\left(t_{1}\right)= & \int_{M \backslash B_{t_{1}}(x, r)} f^{2}\left(t_{1}, y\right) \mu_{t_{1}}(d y) \leq \int_{M} f^{2}\left(t_{1}, y\right) e^{\xi\left(y, t_{1}\right)} \mu_{t_{1}}(d y) \\
\leq & e^{-\left(\Lambda\left(t_{1}\right)-\Lambda\left(t_{2}\right)\right)} \int_{M} f^{2}\left(t_{2}, y\right) e^{\xi\left(y, t_{2}\right)} \mu_{t_{2}}(d y) \\
\leq & e^{-\left(\Lambda\left(t_{1}\right)-\Lambda\left(t_{2}\right)\right)}\left(\int_{B_{t_{2}}(x, \rho)} f^{2}\left(t_{2}, y\right) e^{\xi\left(y, t_{2}\right)} \mu_{t_{2}}(d y)\right. \\
& \left.+\int_{M \backslash B_{t_{2}}(x, \rho)} f^{2}\left(t_{2}, y\right) e^{\xi\left(y, t_{2}\right)} \mu_{t_{2}}(d y)\right) \\
\leq & e^{-\left(\Lambda\left(t_{1}\right)-\Lambda\left(t_{2}\right)\right)}\left(I_{\rho}\left(t_{2}\right)+\int_{B_{t_{2}}(x, \rho)} f^{2}\left(t_{2}, y\right) e^{\xi\left(y, t_{2}\right)} \mu_{t_{2}}(d y)\right) \\
\leq & e^{-\left(\Lambda\left(t_{1}\right)-\Lambda\left(t_{2}\right)\right)}\left(I_{\rho}\left(t_{2}\right)+e^{\frac{-(r-\rho)^{2}}{A\left(t_{0}-t_{2}\right)}} \int_{M} f^{2}\left(t_{2}, y\right) \mu_{t_{2}}(d y)\right.
\end{aligned}
$$

Then remark that the definition of $I_{r}(t)$ is independent of $t_{0}$ and of the corresponding $\xi$, so we can pass to the limit when $t_{0} \searrow t_{1}$ to obtain the desired result.

We apply the above proposition to the heat kernel $P(x, t, y, 0)$ of the equation (3.2) which also satisfies (1.1).

Theorem 4.8. If $\dot{g}(t)=-\operatorname{Ric}_{g(t)}$ for all $t \in\left[0, T_{c}[\right.$ and the following assumptions are satisfied:

- H1: if $M$ is not compact, we suppose that there exists a uniform constant $c_{n}>0$ such that for all $x \in M$ we have $\mu_{0}\left(B_{g(0)}(x, r)\right) \geq$ $c_{n} r^{n}$ (that is a non collapsing condition).

- H2: $\operatorname{Ric}_{g(t)} \geq 0$ for all $t \in\left[0, T_{c}[\right.$.

Then for all $a>1$ there exist two positive explicit constants $q_{a}, m_{a}$ depending only on $a, c_{n}$ and the dimension, such that we have the following heat kernel estimate for all $t \in] 0, T_{c}\left[\right.$ and $x_{0}, y_{0} \in M$ :

$$
P\left(y_{0}, t, x_{0}, 0\right) \leq q_{a} \frac{e^{\int_{0}^{t} \frac{1}{2} \sup _{M} R(u, .)-\frac{1}{4} \inf _{M} R(u, .) d u}}{\left(\mu_{0}\left(B_{0}\left(x_{0}, \sqrt{t}\right)\right)^{\frac{1}{2}} \mu_{0}\left(B_{0}\left(y_{0}, \sqrt{t}\right)\right)^{\frac{1}{2}}\right.} e^{-\frac{m_{a} d_{t}\left(x_{0}, y_{0}\right)^{2}}{16 t}} .
$$

The values of $q_{a}$ and $m_{a}$ are given by (4.3) in the proof. Moreover we could optimize $m_{a}$, in terms of $a>1$, to get a better control for points which are far.

Proof. We could suppose that $\int_{0}^{t} \sup _{M} R(s,) d s<.\infty$ for all $t \in\left[0, T_{c}\right.$ [, else the conclusion is satisfied. Let $f(t, x):=P(x, t, y, 0)$ be the heat kernel of (1.1) that is the solution of equation (3.2). Note that $\mathrm{H} 2$ gives also a condition for non-explosion of the $g(t)$-Brownian motion by Proposition 2.3. 
Then we have by the proof of Theorem 3.1:

$$
\begin{aligned}
\int_{M} f^{2}(t, x) \mu_{t}(d x) & =\int_{M} P^{2}(x, t, y, 0) \mu_{t}(d x) \\
& =\int_{M} P^{* 2}(y, 0, x, t) \mu_{t}(d x) \\
& \leq e \frac{e^{\int_{0}^{t} \tau(u)-\underline{\tau}(u) d u}}{\left(\mu_{0}\left(B_{0}(y, \sqrt{t})\right)\right)} \\
& =e \frac{e^{\frac{1}{2} \int_{0}^{t} \sup _{M} R(u, .)-\inf _{M} R(u, .) d u}}{\left(\mu_{0}\left(B_{0}(y, \sqrt{t})\right)\right)} .
\end{aligned}
$$

Let $0<\rho<r$, and $t_{2}<t_{1}<t_{0}$ then apply Proposition 4.7 to $f(t, x):=$ $P(x, t, y, 0)$ to get :

$$
\begin{aligned}
I_{r}\left(t_{1}\right) & \leq e^{-\left(\Lambda\left(t_{1}\right)-\Lambda\left(t_{2}\right)\right)}\left(I_{\rho}\left(t_{2}\right)+e^{\frac{-(r-\rho)^{2}}{\left(t_{1}-t_{2}\right)}} \int_{M} f^{2}\left(t_{2}, y\right) \mu_{t_{2}}(d y)\right) \\
& \leq e^{-\left(\Lambda\left(t_{1}\right)-\Lambda\left(t_{2}\right)\right)}\left(I_{\rho}\left(t_{2}\right)+e \cdot e^{\frac{-(r-\rho)^{2}}{\left(t_{1}-t_{2}\right)}} \frac{e^{\frac{1}{2} \int_{0}^{t_{2}} \sup _{M} R(u, .)-\inf _{M} R(u, .) d u}}{\left(\mu_{0}\left(B_{0}\left(y, \sqrt{t_{2}}\right)\right)\right)}\right) .
\end{aligned}
$$

Let $a>1$ be a constant. Let us define $r_{k}:=\left(\frac{1}{2}+\frac{1}{k+2}\right) r$ and $t_{k}:=\frac{t}{a^{k}}$.

Thus Proposition 4.7 can be applied to $r_{k+1}<r_{k}$ and $t_{k+1}<t_{k}$, yielding to the same estimate as before :

$$
\begin{aligned}
& I_{r_{k}}\left(t_{k}\right) \\
& \leq e^{-\left(\Lambda\left(t_{k}\right)-\Lambda\left(t_{k+1}\right)\right)}\left(I_{r_{k+1}}\left(t_{k+1}\right)+e \cdot e^{\frac{-\left(r_{k}-r_{k+1}\right)^{2}}{\left(t_{k}-t_{k+1}\right)}} \frac{e^{\frac{1}{2} \int_{0}^{t_{k+1}} \sup _{M} R(u, .)-\inf _{M} R(u, .) d u}}{\left(\mu_{0}\left(B_{0}\left(y, \sqrt{t_{k+1}}\right)\right)\right)}\right) \\
& \leq e^{-\left(\Lambda\left(t_{k}\right)-\Lambda\left(t_{k+1}\right)\right)}\left(I_{r_{k+1}}\left(t_{k+1}\right)+e \cdot e^{\frac{-\left(r_{k}-r_{k+1}\right)^{2}}{\left(t_{k}-t_{k+1}\right)}} \frac{e^{\frac{1}{2} \int_{0}^{t_{0}} \sup _{M} R(u, .)-\inf _{M} R(u, .) d u}}{\left(\mu_{0}\left(B_{0}\left(y, \sqrt{t_{k+1}}\right)\right)\right)}\right) .
\end{aligned}
$$

Applying recursively this inequality, and use $\mathrm{H} 2$ to see that $\Lambda$ is non decreasing, we have for all $k$ :

$$
I_{r_{0}}\left(t_{0}\right) \leq e^{-\left(\Lambda\left(t_{0}\right)-\Lambda\left(t_{k+1}\right)\right)} I_{r_{k+1}}\left(t_{k+1}\right)+e \sum_{i=0}^{k} e^{-\Lambda\left(t_{0}\right)} e^{\frac{-\left(r_{i}-r_{i+1}\right)^{2}}{\left(t_{i}-t_{i+1}\right)}} \frac{e^{\frac{1}{2} \int_{0}^{t_{0}} \sup _{M} R(u, .) d u}}{\left(\mu_{0}\left(B_{0}\left(y, \sqrt{t_{i+1}}\right)\right)\right)}
$$

We also have $\lim _{k \rightarrow \infty} I_{r_{k}}\left(t_{k}\right)=0$ (see Lemma 5.1 in Appendix).

So we can pass to the limit when $k$ goes to infinity in Equation (4.1) to get :

$$
I_{r_{0}}\left(t_{0}\right) \leq e \cdot e^{-\Lambda\left(t_{0}\right)} e^{\frac{1}{2} \int_{0}^{t_{0}} \sup _{M} R(u, .) d u} \sum_{i=0}^{\infty} e^{\frac{-\left(r_{i}-r_{i+1}\right)^{2}}{\left(t_{i}-t_{i+1}\right)}} \frac{1}{\left(\mu_{0}\left(B_{0}\left(y, \sqrt{t_{i+1}}\right)\right)\right)} .
$$


Recall that $r_{i}-r_{i+1}=\frac{r}{(i+3)(i+2)}$ and $t_{i}-t_{i+1}=\frac{t}{a^{i}}\left(1-\frac{1}{a}\right)$. Moreover, by Bishop-Gromov theorem, Theorem 4.19 [13] in the case Ric $\geq 0$ we have

$$
\frac{\mu_{0}\left(B_{0}\left(y, \sqrt{t_{i}}\right)\right)}{\mu_{0}\left(B_{0}\left(y, \sqrt{t_{i+1}}\right)\right)} \leq a^{\frac{n}{2}}:=c_{a} .
$$

Iterating the above inequality we get :

$$
\frac{\mu_{0}\left(B_{0}\left(y, \sqrt{t_{0}}\right)\right)}{\mu_{0}\left(B_{0}\left(y, \sqrt{t_{i+1}}\right)\right)} \leq\left(c_{a}\right)^{i+1}
$$

So we have :

$$
\begin{aligned}
I_{r_{0}}\left(t_{0}\right) & \leq e \frac{e^{-\Lambda\left(t_{0}\right)} e^{\frac{1}{2} \int_{0}^{t_{0}} \sup _{M} R(u, .) d u}}{\mu_{0}\left(B_{0}\left(y, \sqrt{t_{0}}\right)\right)} \sum_{i=0}^{\infty} e^{\frac{-\left(r_{i}-r_{i+1}\right)^{2}}{\left(t_{i}-t_{i+1}\right)}}\left(c_{a}\right)^{i+1} \\
& \leq e \frac{e^{-\Lambda\left(t_{0}\right)} e^{\frac{1}{2} \int_{0}^{t_{0}} \sup _{M} R(u, .) d u}}{\mu_{0}\left(B_{0}\left(y, \sqrt{t_{0}}\right)\right)} \sum_{i=0}^{\infty} e^{\frac{-\left(\frac{r}{(i+3)(i+2)}\right)^{2}}{\left(\frac{t}{\left.a^{i}\left(1-\frac{1}{a}\right)\right)}+(i+1) \log \left(c_{a}\right)\right.}} \\
& \leq e \frac{e^{-\Lambda\left(t_{0}\right)} e^{\frac{1}{2} \int_{0}^{t_{0}} \sup _{M} R(u, .) d u}}{\mu_{0}\left(B_{0}\left(y, \sqrt{t_{0}}\right)\right)} \sum_{i=0}^{\infty} e^{\frac{-a^{i+1} r^{2}}{t_{0}(a-1)(i+3)^{4}}+(i+1) \log \left(c_{a}\right) .}
\end{aligned}
$$

There exists a constant $m_{a}$ such that $\frac{a^{i+1}}{(a-1)(i+3)^{4}} \geq m_{a}(i+2)$, and thus we get :

$$
\begin{aligned}
I_{r_{0}}\left(t_{0}\right) & \leq e \frac{e^{-\Lambda\left(t_{0}\right)} e^{\frac{1}{2} \int_{0}^{t_{0}} \sup _{M} R(u, .) d u}}{\mu_{0}\left(B_{0}\left(y, \sqrt{t_{0}}\right)\right)} \sum_{i=0}^{\infty} e^{\frac{-m_{a} r^{2}}{t_{0}}(i+2)+(i+1) \log \left(c_{a}\right)} \\
& \leq e \frac{e^{-\Lambda\left(t_{0}\right)} e^{\frac{1}{2} \int_{0}^{t_{0}} \sup _{M} R(u, .) d u}}{\mu_{0}\left(B_{0}\left(y, \sqrt{t_{0}}\right)\right)} e^{\frac{-m_{a} r^{2}}{t_{0}}} \sum_{i=0}^{\infty} e^{-(i+1)\left(\frac{m_{a} r^{2}}{t_{0}}-\log \left(c_{a}\right)\right)}
\end{aligned}
$$

If $\frac{m_{a} r^{2}}{t_{0}}-\log \left(c_{a}\right) \geq \log (2)$ then

$$
I_{r_{0}}\left(t_{0}\right) \leq e \frac{e^{-\Lambda\left(t_{0}\right)} e^{\frac{1}{2} \int_{0}^{t_{0}} \sup _{M} R(u, .) d u}}{\mu_{0}\left(B_{0}\left(y, \sqrt{t_{0}}\right)\right)} e^{\frac{-m_{a} r^{2}}{t_{0}}},
$$

If $\frac{m_{a} r^{2}}{t_{0}}-\log \left(c_{a}\right)<\log (2)$ then

$$
\begin{aligned}
I_{r_{0}}\left(t_{0}\right) & \leq \int_{M} P^{2}\left(x, t_{0}, y, 0\right) \mu_{t_{0}}(d x) \\
& =\int_{M} P^{* 2}\left(y, 0, x, t_{0}\right) \mu_{t_{0}}(d x) \\
& \leq e \frac{e^{\frac{1}{2} \int_{0}^{t_{0}} \sup _{M} R(u, .)-\inf _{M} R(u, .) d u}}{\left(\mu_{0}\left(B_{0}\left(y, \sqrt{t_{0}}\right)\right)\right)} \\
& \leq e \frac{e^{\frac{1}{2} \int_{0}^{t_{0}} \sup _{M} R(u, .)-\inf _{M} R(u, .) d u}}{\left(\mu_{0}\left(B_{0}\left(y, \sqrt{t_{0}}\right)\right)\right)} e^{\log (2)+\log \left(c_{a}\right)-\frac{m_{a} r^{2}}{t_{0}}} .
\end{aligned}
$$


We have that for all $a>1$ there exists a constant $q_{a}:=2 e a^{\frac{n}{2}}$ and $\frac{(e \ln a)^{5}}{a^{2}(a-1) 5^{5}} \leq m_{a}$, so we could take in the following $m_{a}=\frac{(e \ln a)^{5}}{a^{2}(a-1) 5^{5}}$ such that :

$$
I_{r}(t) \leq q_{a} \frac{e^{\frac{1}{2} \int_{0}^{t} \sup _{M} R(u, .)-\inf _{M} R(u, .) d u}}{\left(\mu_{0}\left(B_{0}(y, \sqrt{t})\right)\right)} e^{-\frac{m_{a} r^{2}}{t}} .
$$

Case 1 points which are far.

Let $x_{0}, y_{0} \in M$ such that $d_{t}\left(x_{0}, y_{0}\right) \geq \sqrt{t}$, let $r:=\frac{d_{t}\left(x_{0}, y_{0}\right)}{2}$, then by (4.2) (with $I_{r}(t)$ defined with $f(t, x)=P\left(x, t, x_{0}, 0\right)$, there exists $z_{0} \in$ $B_{t}\left(y_{0}, \sqrt{\frac{t}{4}}\right) \subset M \backslash B_{t}\left(x_{0}, r\right)$ such that :

$$
\begin{aligned}
\mu_{t}\left(B_{t}\left(y_{0}, \sqrt{\frac{t}{4}}\right) P^{2}\left(z_{0}, t, x_{0}, 0\right)\right. & \leq I_{r}(t) \\
& \leq q_{a} \frac{e^{\frac{1}{2} \int_{0}^{t} \sup _{M} R(u, .)-\inf _{M} R(u, .) d u}}{\left(\mu_{0}\left(B_{0}\left(x_{0}, \sqrt{t}\right)\right)\right)} e^{-\frac{m_{a} d_{t}\left(x_{0}, y_{0}\right)^{2}}{4 t}} .
\end{aligned}
$$

Then there exists $z_{0} \in B_{t}\left(y_{0}, \sqrt{\frac{t}{4}}\right)$ such that:

$$
P^{2}\left(z_{0}, t, x_{0}, 0\right) \leq q_{a} \frac{e^{\frac{1}{2} \int_{0}^{t} \sup _{M} R(u, .)-\inf _{M} R(u, .) d u}}{\left(\mu_{0}\left(B_{0}\left(x_{0}, \sqrt{t}\right)\right) \mu_{t}\left(B_{t}\left(y_{0}, \sqrt{\frac{t}{4}}\right)\right)\right.} e^{-\frac{m_{a} d_{t}\left(x_{0}, y_{0}\right)^{2}}{4 t}}
$$

By Lemma 4.4 (comparison of volume)

$$
P\left(z_{0}, t, x_{0}, 0\right) \leq\left(q_{a}\right)^{\frac{1}{2}}(\psi(t))^{\frac{1}{2}} \frac{e^{\frac{1}{4} \int_{0}^{t} \sup _{M} R(u, .)-\inf _{M} R(u, .) d u}}{\sqrt{\left(\mu_{0}\left(B_{0}\left(x_{0}, \sqrt{t}\right)\right) \mu_{0}\left(B_{0}\left(y_{0}, \sqrt{\frac{t}{4}}\right)\right)\right)}} e^{-\frac{m_{a} d_{t}\left(x_{0}, y_{0}\right)^{2}}{8 t}},
$$

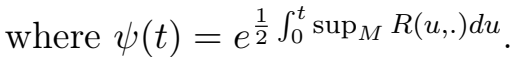

We conclude the proof by using Lemma 4.3 (for $f(t, x):=P\left(x, t, x_{0}, 0\right)$ ) to compare the solution of the heat equation at different points. We have :

$$
\begin{aligned}
P\left(y_{0}, t, x_{0}, 0\right) & \leq \sqrt{P\left(z_{0}, t, x_{0}, 0\right)} \sqrt{\sup _{M} P\left(., \frac{t}{2}, x_{0}, 0\right)} e^{\frac{d_{t}\left(z_{0}, y_{0}\right)^{2}}{t}} \\
& \leq \sqrt{P\left(z_{0}, t, x_{0}, 0\right)} \sqrt{\sup _{M} P\left(., \frac{t}{2}, x_{0}, 0\right)} e^{\frac{1}{4}}
\end{aligned}
$$


Note that using Remark 4.5 and $\mathrm{H} 1$ we have, where $c_{n}$ is the constant coming from H1:

$$
\begin{aligned}
\sup _{M} P\left(., \frac{t}{2}, x_{0}, 0\right) & \leq \frac{e \psi\left(\frac{t}{4}\right)^{\frac{1}{2}} e^{\frac{1}{4} \int_{0}^{\frac{t}{2}}\left(\sup _{M} R(s, .)-\inf _{M} R(s, .)\right) d s}}{\left(\mu_{0}\left(B_{0}\left(x_{0}, \sqrt{\frac{t}{4}}\right)\right)\right)^{\frac{1}{2}} c_{n}^{\frac{1}{2}}\left(\sqrt{\frac{t}{4}}\right)^{\frac{n}{2}}} \\
& \leq \frac{\tilde{c}_{n}^{\frac{1}{2}} e \psi\left(\frac{t}{4}\right)^{\frac{1}{2}} e^{\frac{1}{4} \int_{0}^{\frac{t}{2}}\left(\sup _{M} R(s, .)-\inf _{M} R(s, .)\right) d s}}{\left(\mu_{0}\left(B_{0}\left(x_{0}, \sqrt{\frac{t}{4}}\right)\right)^{\frac{1}{2}} c_{n}^{\frac{1}{2}}\left(\mu_{0}\left(B_{0}\left(y_{0}, \sqrt{\frac{t}{4}}\right)\right)\right)^{\frac{1}{2}}\right.} \\
& \leq q_{n} \frac{\psi(t)^{\frac{1}{2}} e^{\frac{1}{4} \int_{0}^{t}\left(\sup _{M} R(s, .)-\inf _{M} R(s, .)\right) d s}}{\left(\mu_{0}\left(B_{0}\left(x_{0}, \sqrt{t}\right)\right)^{\frac{1}{2}}\left(\mu_{0}\left(B_{0}\left(y_{0}, \sqrt{t}\right)\right)\right)^{\frac{1}{2}}\right.}
\end{aligned}
$$

where in second and last inequality we use Bishop-Gromov theorem to compare volume of ball in positive Ricci curvature case to the corresponding Euclidean volume, i.e. $\left(r \mapsto \frac{\mu(B(x, r))}{\tilde{c}_{n} r^{n}}\right.$ is non increasing and smaller than 1) and $\tilde{c}_{n}$ is the volume of the Euclidean unit ball in dimension $n$, we have $q_{n}=\frac{\tilde{c}_{n}^{\frac{1}{2}} e 2^{n}}{c_{n}^{\frac{1}{2}}}$. Hence we have for $\tilde{q}_{n}=\left(q_{n} 2^{\frac{n}{2}}\right)^{\frac{1}{2}}$ :

$$
P\left(y_{0}, t, x_{0}, 0\right) \leq \tilde{q}_{n}\left(q_{a}\right)^{\frac{1}{4}} \frac{\psi(t)^{\frac{1}{2}} e^{\frac{1}{4} \int_{0}^{t}\left(\sup _{M} R(s, .)-\inf _{M} R(s, .)\right) d s}}{\left(\mu_{0}\left(B_{0}\left(x_{0}, \sqrt{t}\right)\right)\right)^{\frac{1}{2}}\left(\mu_{0}\left(B_{0}\left(y_{0}, \sqrt{t}\right)\right)\right)^{\frac{1}{2}}} e^{-\frac{m_{a} d_{t}\left(x_{0}, y_{0}\right)^{2}}{16 t}} .
$$

Case 2 points are close.

For points $x_{0}, y_{0}$ which are closed that is $d_{t}\left(x_{0}, y_{0}\right) \leq \sqrt{t}$ the above inequality is a consequence of 4.5 since in this case $e^{\frac{-m_{a}}{16}} \leq e^{\frac{-m_{a} d_{t}^{2}\left(x_{0}, y_{0}\right)}{16 t}}$.

Then after changing the function $\psi$ we get :

$$
P\left(y_{0}, t, x_{0}, 0\right) \leq \tilde{q}_{a} \frac{e^{\int_{0}^{t} \frac{1}{2} \sup _{M} R(u, .)-\frac{1}{4} \inf _{M} R(u, .) d u}}{\left(\mu_{0}\left(B_{0}\left(x_{0}, \sqrt{t}\right)\right)^{\frac{1}{2}} \mu_{0}\left(B_{0}\left(y_{0}, \sqrt{t}\right)\right)^{\frac{1}{2}}\right.} e^{-\frac{m_{a} d_{t}\left(x_{0}, y_{0}\right)^{2}}{16 t}}
$$

where

$$
\tilde{q}_{a}=\left(\tilde{q}_{n}\left(2 e a^{\frac{n}{2}}\right)^{\frac{1}{4}}\right) \vee\left(e 2^{\frac{n}{2}} e^{\frac{m_{a}}{16}}\right), m_{a}=\frac{(e \ln a)^{5}}{a^{2}(a-1) 5^{5}}
$$

Proposition 4.9. With the same Hypothesis as in the Theorem 4.8, there exist $c, \lambda>0$ that depend on $n, c_{n}, m_{a}$ such that we get the following lower bound estimate:

$$
c \frac{e^{\int_{0}^{t}-\lambda \sup _{M} R(u, .)+\frac{1}{4} \inf _{M} R(u, .) d u}}{\mu_{0}\left(B _ { 0 } ( z _ { 0 } , \sqrt { t } ) ^ { \frac { 1 } { 2 } } \mu _ { 0 } \left(B_{0}(y, \sqrt{t})^{\frac{1}{2}}\right.\right.} e^{\frac{-4 d_{t}\left(z_{0}, y\right)^{2}}{t}} \leq P\left(z_{0}, t, y, 0\right) .
$$


Proof. We will get the lower bound from the upper bound. Since

$$
\begin{aligned}
\frac{d}{d t} \int_{M} P(x, t, y, 0) \mu_{t}(d x) & =-\frac{1}{2} \int_{M} P(x, t, y, 0) R(t, x) \mu_{t}(d x) \\
& \geq-\frac{1}{2} \sup _{M} R(t, .) \int_{M} P(x, t, y, 0) \mu_{t}(d x)
\end{aligned}
$$

we have

$$
\int_{M} P(x, t, y, 0) \mu_{t}(d x) \geq e^{-\int_{0}^{t} \frac{1}{2} \sup _{M} R(t, .)} .
$$

Let $\beta>0$ large enough, we use Schwarz's inequality :

$$
\begin{aligned}
& \int_{B_{t}(y, \sqrt{\beta t})} P^{2}(x, t, y, 0) \mu_{t}(d x) \geq \frac{1}{\mu_{t}\left(B_{t}(y, \sqrt{\beta t})\right)}\left(\int_{B_{t}(y, \sqrt{\beta t})} P(x, t, y, 0) \mu_{t}(d x)\right)^{2} \\
& =\frac{1}{\mu_{t}\left(B_{t}(y, \sqrt{\beta t})\right)}\left(\int_{M} P(x, t, y, 0) \mu_{t}(d x)-\int_{M \backslash B_{t}(y, \sqrt{\beta t})} P(x, t, y, 0) \mu_{t}(d x)\right)^{2} .
\end{aligned}
$$

We will use the upper bound in Theorem 4.8 and H1 to get an estimate of the last term in the above equation. Let $I(t):=e^{\int_{0}^{t} \frac{1}{2} \sup _{M} R(u, .)-\frac{1}{4} \inf _{M} R(u, .) d u}$

$$
\begin{aligned}
& \int_{M \backslash B_{t}(y, \sqrt{\beta t})} P(x, t, y, 0) \mu_{t}(d x) \leq \frac{q_{a} I(t)}{c_{n} t^{\frac{n}{2}}} \int_{M \backslash B_{t}(y, \sqrt{\beta t})} e^{-\frac{m_{a} d_{t}(x, y)^{2}}{16 t}} \mu_{t}(d x) \\
& \leq \frac{q_{a} I(t)}{c_{n} t^{\frac{n}{2}}} \int_{M \backslash B_{t}(y, \sqrt{\beta t})} e^{-\frac{m_{a} d_{t}(x, y)^{2}}{32 t}} \mu_{t}(d x) e^{-\frac{m_{a} \beta}{32}} \\
& \leq \frac{q_{a} I(t) e^{-\frac{m_{a} \beta}{32}}}{c_{n} t^{\frac{n}{2}}} \sum_{k=1}^{\infty} \int_{B_{t}\left(y, 2^{k} \sqrt{\beta t}\right) \backslash B_{t}\left(y, 2^{k-1} \sqrt{\beta t}\right)} e^{-\frac{m_{a} d_{t}(x, y)^{2}}{32 t}} \mu_{t}(d x) \\
& \leq \frac{q_{a} I(t) e^{-\frac{m_{a} \beta}{32}}}{c_{n} t^{\frac{n}{2}}} \sum_{k=1}^{\infty} e^{-\frac{\left.m_{a} 2^{2(k-1)}\right)_{\beta}}{32}} \mu_{t}\left(B_{t}\left(y, 2^{k} \sqrt{\beta t}\right)\right) \\
& \leq \frac{q_{a} I(t) e^{-\frac{m_{a} \beta}{32}}}{c_{n}} \sum_{k=1}^{\infty} e^{-\frac{m_{a} 2^{2(k-1)} \beta}{32}} \tilde{c}_{n}\left(2^{k} \sqrt{\beta}\right)^{n},
\end{aligned}
$$

where use $\mathrm{H} 2$ and the volume comparison theorem in the last inequality. Let $\beta=2\left(\frac{1}{2} \int_{0}^{t} \sup _{M} R(u,) d u+C.\right) /\left(m_{a} / 32\right)$, since for all $k \geq 0,2^{2(k)} \geq k+1$ and $R(u,) \geq$.0 :

$$
\begin{aligned}
& I(t) \sum_{k=1}^{\infty} e^{-\frac{m_{a} 2^{2(k-1)} \beta}{32}}\left(2^{k} \sqrt{\beta}\right)^{n} \leq I(t) 2^{n} \beta^{\frac{n}{2}} \sum_{k=0}^{\infty} e^{-\frac{m_{a}(k+1) \beta}{32}} 2^{n k} \\
& \leq I(t) \beta^{\frac{n}{2}} e^{-\frac{m_{a} \beta}{32}} \frac{2^{n}}{1-2^{n}\left(e^{-\frac{m_{a} \beta}{32}}\right)} \\
& \leq e^{-\frac{1}{2} \int_{0}^{t} \sup _{M} R(u, .) d u-C} \beta^{\frac{n}{2}} \frac{2^{n}}{1-2^{n}\left(e^{-\frac{m_{a} \beta}{32}}\right)}
\end{aligned}
$$


The last term goes to 0 as $C$ tends to infinity. Hence for a constant $C$ large enough (that only depends on $n, c_{n}, q_{a}$ and $m_{a}$ ) and independent on $t$, we have:

$$
\int_{M \backslash B_{t}(y, \sqrt{\beta t})} P(x, t, y, 0) \mu_{t}(d x) \leq \frac{1}{2} e^{-\frac{m_{a} \beta}{32}} \leq \frac{1}{2} e^{-\frac{1}{2} \int_{0}^{t} \sup _{M} R(u, .) d u} .
$$

Thus

$$
\int_{B_{t}(y, \sqrt{\beta t})} P^{2}(x, t, y, 0) \mu_{t}(d x) \geq \frac{1}{4 \mu_{t}\left(B_{t}(y, \sqrt{\beta t})\right)}\left(e^{-\frac{1}{2} \int_{0}^{t} \sup _{M} R(u, .) d u}\right)^{2} .
$$

Hence there exist $x_{1} \in B_{t}(y, \sqrt{\beta t})$ such that :

$$
P\left(x_{1}, t, y, 0\right) \geq \frac{1}{2 \mu_{t}\left(B_{t}(y, \sqrt{\beta t})\right)}\left(e^{-\frac{1}{2} \int_{0}^{t} \sup _{M} R(u, .) d u}\right)
$$

Using the volume comparison theorem, Hypotheses H2 and H1, we have :

$$
\mu_{t}\left(B_{t}(y, \sqrt{\beta t})\right) \leq \tilde{c}_{n} \beta^{\frac{n}{2}} t^{\frac{n}{2}} \leq \frac{\tilde{c}_{n}}{c_{n}} \beta^{\frac{n}{2}} \mu_{0}\left(B_{0}(y, \sqrt{t})\right)
$$

so there exists a constant cst that depends on the dimension and the constant $c_{n}$ of $\mathrm{H} 1$

$$
P\left(x_{1}, t, y, 0\right) \geq \operatorname{cst} \frac{1}{\mu_{0}\left(B_{0}(y, \sqrt{t})\right)} \frac{e^{-\frac{1}{2} \int_{0}^{t} \sup _{M} R(u, .) d u}}{\left.\left(\int_{0}^{t} \sup _{M} R(u, .) d u+2 C\right) /\left(m_{a} / 32\right)\right)^{\frac{n}{2}}} .
$$

Since for all $x \geq 0$ we have

$$
\frac{e^{-\frac{x}{2}}}{(x+2 C)^{\frac{n}{2}}} \geq \frac{e^{-\left(\frac{1}{2}+\frac{n}{4 C}\right) x}}{(2 C)^{\frac{n}{2}}}
$$

we get for a $\operatorname{cst}\left(n, c_{n}, m_{a}\right)$ that can change from a line to line and using H2 :

$$
P\left(x_{1}, t, y, 0\right) \geq \operatorname{cst} \frac{e^{-\left(\frac{1}{2}+\frac{n}{4 C}\right) \int_{0}^{t} \sup _{M} R(u, .) d u}}{\mu_{0}\left(B_{0}(y, \sqrt{t})\right)} \geq \operatorname{cst} \frac{e^{-\left(\frac{1}{2}+\frac{n}{4 C}\right) \int_{0}^{t} \sup _{M} R(u, .) d u}}{t^{\frac{n}{2}}} .
$$

We conclude the proof by using lemma 4.3 (for $f(t, x):=P(x, t, y, 0)$ ) to compare the solution of the heat equation at different points. We have for all $z_{0}$ :

$$
P\left(x_{1}, t, y, 0\right) \leq \sqrt{P\left(z_{0}, t, y, 0\right)} \sqrt{\sup _{M} P\left(., \frac{t}{2}, y, 0\right)} e^{\frac{d_{t}\left(z_{0}, x_{1}\right)^{2}}{t}}
$$

With the triangle inequality

$$
d_{t}\left(z_{0}, x_{1}\right)^{2} \leq 2 d_{t}\left(z_{0}, y\right)^{2}+2 \beta t
$$

and so

$$
P\left(x_{1}, t, y, 0\right) \leq \sqrt{P\left(z_{0}, t, y, 0\right)} \sqrt{\sup _{M} P\left(., \frac{t}{2}, y, 0\right)} e^{\frac{2 d_{t}\left(z_{0}, y\right)^{2}}{t}} e^{2 \beta} .
$$


As in the proof of Theorem 4.8 and using $\mathrm{H} 1$ we get:

$$
\begin{aligned}
\sup _{M} P\left(., \frac{t}{2}, y, 0\right) & \leq \operatorname{cst} \frac{e^{\int_{0}^{t}\left(\frac{1}{2} \sup _{M} R(s, .)-\frac{1}{4} \inf _{M} R(s, .)\right) d s}}{\left(\mu_{0}\left(B_{0}\left(z_{0}, \sqrt{t}\right)\right)^{\frac{1}{2}}\left(\mu_{0}\left(B_{0}(y, \sqrt{t})\right)\right)^{\frac{1}{2}}\right.} \\
& \leq \operatorname{cst} \frac{e^{\int_{0}^{t}\left(\frac{1}{2} \sup _{M} R(s, .)-\frac{1}{4} \inf _{M} R(s, .)\right) d s}}{t^{\frac{n}{2}}} .
\end{aligned}
$$

Hence :

$$
\begin{aligned}
\operatorname{cst} \frac{e^{-\left(\frac{1}{2}+\frac{n}{4 C}\right) \int_{0}^{t} \sup _{M} R(u, .) d u}}{t^{\frac{n}{2}}} \leq P\left(x_{1}, t, y, 0\right) \\
\leq \sqrt{P\left(z_{0}, t, y, 0\right)} \sqrt{\sup _{M} P\left(., \frac{t}{2}, y, 0\right) e^{\frac{2 d_{t}\left(z_{0}, y\right)^{2}}{t}} e^{2 \beta} .}
\end{aligned}
$$

Thus

$$
\begin{aligned}
& \operatorname{cst} \frac{e^{-\left(\frac{1}{2}+\frac{n}{4 C}\right) \int_{0}^{t} \sup _{M} R(u, .) d u}}{t^{\frac{n}{2}}} \\
& \leq \sqrt{P\left(z_{0}, t, y, 0\right)} \frac{e^{\int_{0}^{t}\left(\frac{1}{4} \sup _{M} R(s, .)-\frac{1}{8} \inf _{M} R(s, .)\right) d s}}{t^{\frac{n}{4}}} e^{\frac{2 d_{t}\left(z_{0}, y\right)^{2}}{t}} e^{2 \beta}
\end{aligned}
$$

We recall the definition of $\beta$ there exist some constants $c, \lambda>0$ that depend on $n, c_{n}, m_{a}$ such that :

$$
c \frac{e^{\int_{0}^{t}-\lambda \sup _{M} R(u, .)+\frac{1}{8} \inf _{M} R(u, .) d u}}{t^{\frac{n}{4}}} e^{\frac{-2 d_{t}\left(z_{0}, y\right)^{2}}{t}} \leq \sqrt{P\left(z_{0}, t, y, 0\right)} .
$$

After using $\mathrm{H} 1$ again for a constant that could change from line to line, we have :

$$
c \frac{e^{\int_{0}^{t}-\lambda \sup _{M} R(u, .)+\frac{1}{4} \inf _{M} R(u, .) d u}}{\mu_{0}\left(B _ { 0 } ( z _ { 0 } , \sqrt { t } ) ^ { \frac { 1 } { 2 } } \mu _ { 0 } \left(B_{0}(y, \sqrt{t})^{\frac{1}{2}}\right.\right.} e^{\frac{-4 d_{t}\left(z_{0}, y\right)^{2}}{t}} \leq P\left(z_{0}, t, y, 0\right),
$$

which is the desired lower bound.

Remark 4.10. The constants $\frac{1}{2}$ and $\frac{1}{4}$ are far from being optimal in Theorem 4.8. Hypothesis $\mathrm{H} 1$ and $\mathrm{H} 2$ are used many time to compare the volume of some ball and the Euclidean one's.

Note that for manifold of dimension three, $\mathrm{H} 2$ reduce to $\mathrm{Ric}_{g(0)} \geq 0$ e.g. in Corollary 9.2 [18] and page 193 in [8].

The existence of Ricci flow for a complete non-compact manifold at least for short time have been proved in [24] Theorem 2.1, under boundedness conditions for the Riemannian tensor of $(M, g(0))$. There also have some uniqueness results in this direction in [6] Theorem 1.1. 


\section{Appendix}

Lemma 5.1. With the same hypothesis as in Theorem 4.8 and suppose $\int_{0}^{t} \sup _{M} R(s,) d s<.\infty$ for all $t \in\left[0, T_{c}\left[\right.\right.$. Let $a>1$ be a constant, $r_{k}:=$ $\left(\frac{1}{2}+\frac{1}{k+2}\right) r$ and $t_{k}:=\frac{t}{a^{k}}$. We have

$$
\lim _{k \longrightarrow \infty} I_{r_{k}}\left(t_{k}\right)=0
$$

Proof. We use H1 and $\int_{0}^{t} \sup _{M} R(s,) d s<.\infty$ to get a global polynomial bound of the heat kernel i.e. Remark 4.5. We obtain that for all $t \leq \frac{T_{c}}{2}$ :

$$
\begin{aligned}
P(x, t, y, 0) & \leq \frac{C s t}{\left.\left(\mu_{0}\left(B_{0}\left(x, \sqrt{\frac{t}{2}}\right)\right)\right)^{\frac{1}{2}} \mu_{0}\left(B_{0}\left(y, \sqrt{\frac{t}{2}}\right)\right)\right)^{\frac{1}{2}}} \\
& \leq \frac{c s t}{t^{\frac{n}{2}}} .
\end{aligned}
$$

Then for a constant which could change from line to line :

$$
\begin{aligned}
I_{r}(t) & =\int_{\left(M \backslash B_{t}(y, r)\right)} P^{2}(x, t, y, 0) \mu_{t}(d x) \leq \frac{c s t}{t^{\frac{n}{2}}} \int_{\left(M \backslash B_{t}(y, r)\right)} P(x, t, y, 0) \mu_{t}(d x) \\
& \leq \frac{c s t}{t^{\frac{n}{2}}} \int_{\left(M \backslash B_{t}(y, r)\right)} P^{*}(y, 0, x, t) \mu_{t}(d x) \\
& \leq \frac{c s t}{t^{\frac{n}{2}}} \int_{\left(M \backslash B_{t}(y, r)\right)} \bar{P}(y, t, x, t) \mu_{t}(d x) \\
& \leq c s t \frac{\mathbb{P}_{y}\left(\tau_{r}<t\right)}{t^{\frac{n}{2}}}
\end{aligned}
$$

where in $\tau_{r}:=\inf \left\{t>0, d_{t}\left(X_{t}(y), y\right)=r\right\}$, and $X_{t}(y)$ is a $g(t)$-Brownian motion started at $y$, note that $X_{t}(y)$ does not explode using $\mathrm{H} 2$ and Proposition 2.3 .

Let $\rho_{t}:=d_{t}\left(X_{t}(y), y\right)$, use $(2.3)$ and Itô's formula we get for the real Brownian motion $b_{t}:=\int_{0}^{t} \mathbb{1}_{X_{s} \notin C u t_{s}(y)}\left\langle\nabla^{s} d_{s}\left(y, X_{s}\right), d^{\nabla_{s}} X_{s}\right\rangle_{g(s)}$

$$
\begin{aligned}
d \rho_{t}^{2} & =2 \rho_{t} d \rho_{t}+d t \\
& \leq 2 \rho_{t}\left(\mathbb{1}_{X_{t} \notin C u t_{t}\left(x_{0}\right)}\left(\frac{1}{2} \Delta_{g(t)} d_{t}(y, .)+\frac{\partial d_{t}(y, .)}{\partial t}\right)\left(X_{t}\right)\right) d t+d t \\
& +2 \rho_{t} d b_{t} .
\end{aligned}
$$

By the Laplacian comparison theorem (Theorem 3.4.2 in [19]) we also have, since by $\mathrm{H} 2, \operatorname{Ric}_{g(t)} \geq 0$ for all $t \in\left[0, T_{c}\right.$, within the cutlocus:

$$
\Delta_{g(t)} d_{t}(y, .) \leq \frac{n-1}{d_{t}(y, .)} \quad \text { and } \quad \frac{\partial d_{t}(y, .)}{\partial t} \leq 0 .
$$

Hence

$$
d \rho_{t}^{2} \leq n d t+2 \rho_{t} d b_{t}
$$


at time $t=\tau_{r}$,

$$
r^{2} \leq n \tau_{r}+\int_{0}^{\tau_{r}} 2 \rho_{t} d b_{t}
$$

On the event $\left\{\tau_{r} \leq t\right\}$,

$$
\frac{r^{2}-n t}{2} \leq \int_{0}^{\tau_{r}} \rho_{t} d b_{t}
$$

Note that $\int_{0}^{\tau_{r}} \rho_{t} d b_{t}=W_{T}$, where $W$ is a Brownian motion and $T=\int_{0}^{\tau_{r}} \rho_{s}^{2} d s$. On the event $\left\{\tau_{r} \leq t\right\}$ we also have $T \leq r^{2} t$. Then the event $\left\{\tau_{r} \leq t\right\}$ implies

$$
\frac{r^{2}-n t}{2} \leq W_{T} \leq \sup _{s \in\left[0, r^{2} t\right]} W_{s} \sim r \sqrt{t}\left|W_{1}\right|,
$$

hence for $r^{2}-n t>0$,

$$
\begin{aligned}
\mathbb{P}_{y}\left(\tau_{r}<t\right) & \leq \mathbb{P}_{0}\left(\frac{r^{2}-n t}{2 r \sqrt{t}} \leq\left|W_{1}\right|\right) \\
& \leq \frac{2}{\sqrt{2 \pi}\left(\frac{r^{2}-n t}{2 r \sqrt{t}}\right)} e^{-\left(\frac{r^{2}-n t}{2 r \sqrt{t}}\right)^{2}} .
\end{aligned}
$$

Since $a>1$ be a constant $r_{k}:=\left(\frac{1}{2}+\frac{1}{k+2}\right) r$ and $t_{k}:=\frac{t}{a^{k}}$, we have

$$
\frac{\mathbb{P}_{y}\left(\tau_{r_{k}}<t_{k}\right)}{t_{k}^{\frac{n}{2}}} \rightarrow_{k \rightarrow \infty} 0
$$

and then

$$
\lim _{k \longrightarrow \infty} I_{r_{k}}\left(t_{k}\right)=0
$$

\section{REFERENCES}

[1] Marc Arnaudon, Kolehe Abdoulaye Coulibaly, and Anton Thalmaier. Brownian motion with respect to a metric depending on time: definition, existence and applications to Ricci flow. C. R. Math. Acad. Sci. Paris, 346(13-14):773-778, 2008.

[2] Marc Arnaudon, Koléhè Abdoulaye Coulibaly, and Anton Thalmaier. Horizontal diffusion in $C^{1}$ path space. In Séminaire de Probabilités XLIII, volume 2006 of Lecture Notes in Math., pages 73-94. Springer, Berlin, 2011.

[3] Marc Arnaudon, Anton Thalmaier, and Feng-Yu Wang. Harnack inequality and heat kernel estimates on manifolds with curvature unbounded below. Bull. Sci. Math., 130(3):223-233, 2006.

[4] Dominique Bakry, Ivan Gentil, and Michel Ledoux. On Harnack inequalities and optimal transportation. Ann. Sc. Norm. Super. Pisa Cl. Sci. (5), 14(3):705-727, 2015.

[5] Xiaodong Cao and Qi S. Zhang. The conjugate heat equation and ancient solutions of the Ricci flow. Adv. Math., 228(5):2891-2919, 2011.

[6] Bing-Long Chen and Xi-Ping Zhu. Uniqueness of the Ricci flow on complete noncompact manifolds. J. Differential Geom., 74(1):119-154, 2006.

[7] Li-Juan Cheng. Diffusion semigroup on manifolds with time-dependent metrics. Forum Math., 29(4):775-798, 2017. 
[8] Bennett Chow, Sun-Chin Chu, David Glickenstein, Christine Guenther, James Isenberg, Tom Ivey, Dan Knopf, Peng Lu, Feng Luo, and Lei Ni. The Ricci flow: techniques and applications. Part II, volume 144 of Mathematical Surveys and Monographs. American Mathematical Society, Providence, RI, 2008. Analytic aspects.

[9] Bennett Chow, Sun-Chin Chu, David Glickenstein, Christine Guenther, James Isenberg, Tom Ivey, Dan Knopf, Peng Lu, Feng Luo, and Lei Ni. The Ricci flow: techniques and applications. Part III. Geometric-analytic aspects, volume 163 of Mathematical Surveys and Monographs. American Mathematical Society, Providence, RI, 2010.

[10] Koléhè A. Coulibaly-Pasquier. Brownian motion with respect to time-changing Riemannian metrics, applications to Ricci flow. Ann. Inst. Henri Poincaré Probab. Stat., 47(2):515-538, 2011.

[11] Koléhè A. Coulibaly-Pasquier. Some stochastic process without birth, linked to the mean curvature flow. Ann. Probab., 39(4):1305-1331, 2011.

[12] M. Gage and R. S. Hamilton. The heat equation shrinking convex plane curves. $J$. Differential Geom., 23(1):69-96, 1986.

[13] Sylvestre Gallot, Dominique Hulin, and Jacques Lafontaine. Riemannian geometry. Universitext. Springer-Verlag, Berlin, third edition, 2004.

[14] Fu-Zhou Gong and Feng-Yu Wang. Heat kernel estimates with application to compactness of manifolds. Q. J. Math., 52(2):171-180, 2001.

[15] Alexander Grigor' yan. Integral maximum principle and its applications. Proc. Roy. Soc. Edinburgh Sect. A, 124(2):353-362, 1994.

[16] Alexander Grigor' yan. Gaussian upper bounds for the heat kernel on arbitrary manifolds. J. Differential Geom., 45(1):33-52, 1997.

[17] Christine M. Guenther. The fundamental solution on manifolds with time-dependent metrics. J. Geom. Anal., 12(3):425-436, 2002.

[18] Richard S. Hamilton. Three-manifolds with positive Ricci curvature. J. Differential Geom., 17(2):255-306, 1982.

[19] Elton P. Hsu. Stochastic analysis on manifolds, volume 38 of Graduate Studies in Mathematics. American Mathematical Society, Providence, RI, 2002.

[20] Kazumasa Kuwada and Robert Philipowski. Non-explosion of diffusion processes on manifolds with time-dependent metric. Math. Z., 268(3-4):979-991, 2011.

[21] Peter Li and Shing-Tung Yau. On the parabolic kernel of the Schrödinger operator. Acta Math., 156(3-4):153-201, 1986.

[22] Robert J. McCann and Peter M. Topping. Ricci flow, entropy and optimal transportation. Amer. J. Math., 132(3):711-730, 2010.

[23] Lei Ni. Ricci flow and nonnegativity of sectional curvature. Math. Res. Lett., 11(56):883-904, 2004.

[24] Wan-Xiong Shi. Deforming the metric on complete Riemannian manifolds. J. Differential Geom., 30(1):223-301, 1989.

[25] Feng-Yu Wang. Logarithmic Sobolev inequalities on noncompact Riemannian manifolds. Probab. Theory Related Fields, 109(3):417-424, 1997.

Institut Élie Cartan de Lorraine, UMR 7502

UNIVERSITÉ DE LORRAINE AND CNRS

E-mail address: kolehe.coulibaly@univ-lorraine.fr 